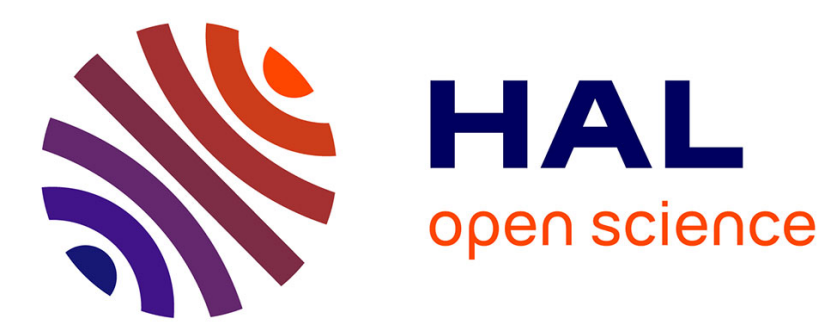

\title{
A phase-field method for propagating fluid-filled fractures coupled to a surrounding porous medium
}

\author{
Andro Mikelic, M.F. Wheeler, Thomas Wick
}

\section{To cite this version:}

Andro Mikelic, M.F. Wheeler, Thomas Wick. A phase-field method for propagating fluid-filled fractures coupled to a surrounding porous medium. Multiscale Modeling and Simulation: A SIAM Interdisciplinary Journal, 2015, 13 (1), pp.367-398. 10.1137/140967118 . hal-01114228

\section{HAL Id: hal-01114228 \\ https://hal.science/hal-01114228}

Submitted on 9 Feb 2015

HAL is a multi-disciplinary open access archive for the deposit and dissemination of scientific research documents, whether they are published or not. The documents may come from teaching and research institutions in France or abroad, or from public or private research centers.
L'archive ouverte pluridisciplinaire HAL, est destinée au dépôt et à la diffusion de documents scientifiques de niveau recherche, publiés ou non, émanant des établissements d'enseignement et de recherche français ou étrangers, des laboratoires publics ou privés. 


\title{
A phase-field method for propagating fluid-filled fractures coupled to a surrounding porous medium
}

\author{
Andro Mikelić * \\ Mary F. Wheeler ${ }^{\dagger}$ \\ Thomas Wick $\ddagger$
}

The recently introduced phase-field approach for pressurized fractures in a porous medium offers various attractive computational features for numerical simulations of cracks such as joining, branching, and non-planar propagation in possibly heterogeneous media. In this study, the pressurized phase-field framework is extended to fluid-filled fractures in which the pressure is computed from a generalized parabolic diffraction problem. Here, the phase-field variable is used as indicator function to combine reservoir and fracture pressure. The resulting three-field framework (elasticity, phase-field, pressure) is a multiscale problem that is based on the Biot equations. The proposed numerical solution algorithm iteratively decouples the equations using a fixed-stress splitting. The framework is substantiated with several numerical benchmark tests in two- and three dimensions.

Keywords: finite elements; phase-field; Biot system; fixed-stress iterative coupling; fracture propagation

\section{Introduction}

Presently, crack propagation in elastic and porous media is one of the major research topics in mechanical, energy and environmental engineering. Of particular interest is induced hydraulic fracturing or hydrofracturing in subsurface modeling. This is a technique used to release petroleum and natural gas that includes shale gas, tight gas, and coal seam gas for extraction. Fracking creates fractures from a wellbore drilled into reservoir rock formations. There are economic benefits of extracting vast amounts of formerly inaccessible hydrocarbons. In addition, there are environmental benefits of producing natural gas, much of which is produced in the United States from fracking making the country nearly energy-independent. Opponents to fracking point to environmental impacts such as contamination of ground water, risks to air quality, migration of fracturing chemical and surface contamination from spills, to name a few. For this reason, hydraulic fracturing is being heavily scrutinized. This results in the need for accurate and robust mathematical and computational models for treating fluid field fractures surrounded by a poroelastic medium.

\footnotetext{
${ }^{*}$ Université de Lyon, CNRS UMR 5208, Université Lyon 1, Institut Camille Jordan, 43, blvd. du 11 novembre 1918, 69622 Villeurbanne Cedex, France

${ }^{\dagger}$ Center for Subsurface Modeling, The Institute for Computational Engineering and Sciences, The University of Texas at Austin, Austin, Texas 78712, USA

${ }^{\ddagger}$ Center for Subsurface Modeling, The Institute for Computational Engineering and Sciences, The University of Texas at Austin, Austin, Texas 78712, USA
} 
In principle, we deal with equations describing the porous medium which are based on Biot's system [2-4] and on the other hand, we need a framework for the description of pressurized and natural fractures. The combination of both settings leads to a challenging multiscale problem where only few results have been published to date. For fracture descriptions and crack propagation, we use a fixed-topology phase-field approach that remodels Griffith's [25] criterium for brittle fracture [5, 7-10, 20]. Here, the lower-dimensional crack surface is approximated by a phase-field function. This introduces a diffusive transition zone (brittle zone or mushy-zone are also common expressions depending upon the discipline) between the broken and the unbroken material; see Figure 1.

In this paper, we are specifically interested in fluid-filled fractures and their propagation in which two pressure equations are coupled to a single model. Moreover, the reservoir zone is modeled as a linear elastic material $[17,40]$. Particular interest of such a modeling is in dam constructions, damage in blood arteries where blood enters into the tissue, or processes in subsurface modeling. In the last years, several methods for pressurized fracture and crack propagation have been proposed. Worthy to mention are the studies in [33] using an implicit moving mesh algorithm; [41], employing a moving-mesh approach with local grid refinement; a special zerothickness finite element approach [11]; treating pressurized fractures with a boundary element method in a reservoir [17, 23]; and the partition of unity and extended finite element approaches [26, 29] handling fluid flow in a fracture. Another method of recent research is peridynamics that is employed to study pressurized and fluid-filled fractures [30]. To the best of our knowledge, the previously mentioned phase-field approach was first applied to pressurized cracks in [6], and a rigorous model investigation was first undertaken in [35, 36]. In these models, the irreversibility condition for crack growth is of crucial importance. An efficient implementation and verification of this constraint based on the augmented Lagrangian method is investigated in [46].
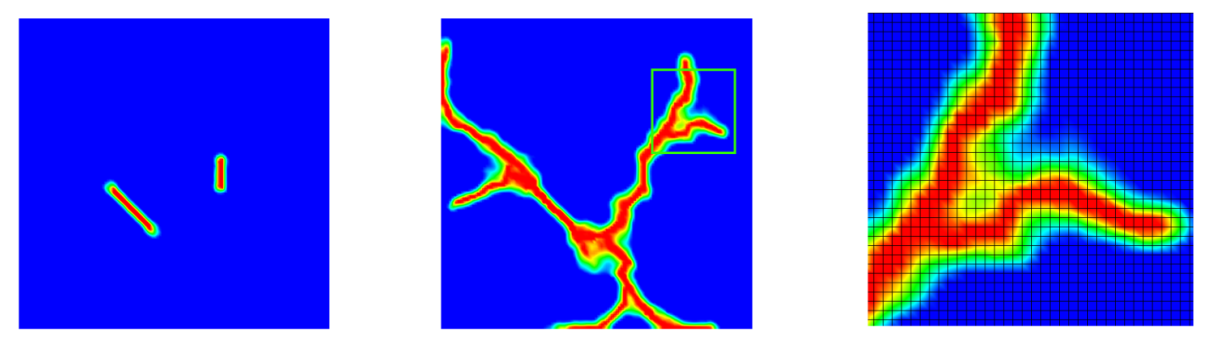

Figure 1: Explication of the fixed-topology finite element phase-field approach: Lower-dimensional cracks are approximated with the help of a phase-field function. The phase-field is an indicator function with values 0 in the crack (red) and 1 in the unbroken zone (blue). The mushy-zone provides a smooth interpolation between 0 and 1 indicated in green color. The middle figure shows the evolution of these two cracks at the final time. Here, we observe the major advantages of this approach such as joining, branching and nonplanar crack growth in heterogeneous media. The right subfigure focuses on the branching in the green square. Moreover, the fixed grid is shown illustrating that no grid adaptation is applied in the phase-field method. The figures are extensions of our numerical results shown in Section 4 using heterogenous geomechanical parameters.

The major advantages of using phase-field modeling for crack propagation are four-fold. First, it is a fixedtopology approach in which remeshing is avoided. Second, the model is purely based on energy minimization and crack nucleation, propagation and the path are automatically determined (avoiding postprocessing of certain quantities such as stress intensity factors). Third, joining and branching of (multiple) cracks do not require any additional techniques. Consequently, it allows simple handling of large and complex fracture networks in 
heterogeneous media as illustrated in Figure 1. It is important to notice that quantities of interest such as the crack opening displacement (the aperture) can be recovered with the help of the phase-field function or (as shown in this work) it can be used as indicator variable to distinguish different time-dependent domains.

In the following, we briefly describe the underlying equations and their coupling. Our model consists of the fixed stress split of the Biot equations, with the phase field regularization in the mechanics part, which has been introduced in [35, 36]. In this splitting, we first solve the "fixed stress" [38, 42] for the pressure. The equations are posed in the poroelastic reservoir $\Omega_{R}(t)$ and in the fracture $\Omega_{F}(t)$. On $\Gamma(t)=\bar{\Omega}_{R}(t) \cap \bar{\Omega}_{F}(t)$ we impose interface conditions. $\mathrm{T}$ hen, we have

$$
\begin{gathered}
\vartheta_{j} \partial_{t} p_{j}+\operatorname{div}\left(\frac{K_{j}}{\eta_{j}}\left(\rho_{j}^{0} g-\nabla p_{j}\right)\right)=\frac{q_{j}}{\rho_{j}^{0}} \quad \text { in } \quad \Omega_{j}(t), j=R, F, \\
\frac{K_{R}}{\eta_{R}}\left(\rho_{R}^{0} g-\nabla p_{R}\right) \cdot n=\frac{K_{F}}{\eta_{F}}\left(\rho_{F}^{0} g-\nabla p_{F}\right) \cdot n \quad \text { and } \quad p_{R}=p_{F} \quad \text { on } \Gamma(t) .
\end{gathered}
$$

Next, the calculated pressures are used to solve the mechanics part of the "fixed stress" system. This system is modified (as described in [35, 37]) and includes the phase field characterization of the fluid-filled pressurized fracture.

$$
\begin{gathered}
-\operatorname{div}\left\{\left((1-\kappa) \varphi_{+}^{2}+\kappa\right) \mathcal{G} e(u)\right\}+\varphi_{+}^{2} \nabla p+(\alpha-1) \nabla\left(\varphi_{+}^{2} p\right)=0 \\
\text { in } \quad \Omega=\Omega_{R}(t) \cup \Gamma(t) \cup \Omega_{F}(t),
\end{gathered}
$$

We finally introduce the phase-field equation:

$$
\begin{gathered}
\mathcal{L}(u, \varphi)=-G_{c} \varepsilon \Delta \varphi-\frac{G_{c}}{\varepsilon}(1-\varphi)+(1-\kappa) \varphi_{+}^{2} \mathcal{G} e(u): e(u)+ \\
2(1-\alpha) \varphi_{+} p \operatorname{div} u+2 \varphi_{+} \nabla p \cdot u \leq 0 \quad \text { in } \Omega \\
\partial_{t} \varphi \leq 0 \quad \text { in } \quad \Omega, \quad \partial_{t} \varphi \cdot \mathcal{L}(u, \varphi)=0 \quad \text { in } \Omega
\end{gathered}
$$

We recall that $\mathcal{G}$ is Gassmann's elasticity tensor, characterized by Lamé's constants $\lambda$ and $\mu, \alpha(\approx 1)$ is Biot's parameter, $G_{c}$ is the fracture toughness and $\varepsilon$ and $\kappa, \kappa \ll \varepsilon$ are small positive parameters. The calculated phase field $\varphi$ is used to update the fracture domain $\Omega_{F}(t)$ and the globally defined displacement field $u$ is employed to update $q_{R}$.

In our proposed solution algorithm, we then go back to equations (1)-(2); solving again for the pressures and continuing with the iterative process. Equations (1)-(2) for the pressures are introduced and discussed in detail in Sec 2. A special attention is brought to the explanation of the model (of the coefficients and of the interface conditions). The fracture domain is considered as a 3D domain and in Remark 2.2 we give details on the permeability interpolation. Equations (3)-(5) are stated in subsection 2.5 in their time discretized (incremental) form. The constraint $\partial_{t} \varphi \leq 0$ is handled using penalization. Detailed explanation of the equations are provided in [35-37].

As we see from the previous equations, geomechanics and reservoir flow are coupled through volume terms through the fixed-stress iteration. Reservoir pressure and fracture pressure are coupled through an interface law and in addition, an interface law is employed to couple fracture pressure to geomechanics and phase-field (a detailed derivation can be found in [35, 37]).

Summarizing and with regard to the existing studies presented in $[36,46]$, the present paper presents two novelties:

- Combining the full Biot system with phase-field modeling; namely the fixed (synthetic) pressure used in 
$[36,46]$ is replaced by the Biot pressure. This is a major extension resulting in a multiscale formulation;

- We introduce a weighted pressure diffraction formulation in which the phase-field variable is used as indicator function determining the location of the fracture;

The numerical discretization is based on an incremental version $[36,46]$. In particular, a Galerkin finite element scheme is used for spatial discretization. Our solution algorithm is based on the fixed-stress split [38] in which the equations are solved in a decoupled fashion. That is, we have an additional outer loop arising from the augmented Lagrangian penalization, we solve in the following way:

- On the inner level, the fixed-stress iteration is employed to solve the pressure-elasticity problem. In particular the fixed-stress splitting allows for the possibility of sophisticated solvers for the linear system;

- In the outer augmented Lagrangian loop, we solve iteratively to satisfy the irreversibility constraint of crack growth;

- Then we continue the next time step;

- The nonlinear phase-field equation is solved with Newton's method whereas the two other problems are linear.

Of particular interest is the interaction and discretization of the pressure equations. In most studies the fracture flow is computed with a lower-dimensional lubrication equation) [11, 29, 41]. However, [44, 45] and [27] introduced a coupling of reservoir and fracture pressure formulated in the same dimension. They define a fracture porosity to compute pressure in those cells (i.e., grid blocks) which are (partially) occupied by the fracture. In our work, we apply the phase-field variable as an indicator function to differentiate between the equations. It is established in [36] that the phase-field variable is a smooth function, which avoids well-known difficulties in numerical quadrature. In [27] a direction-dependent permeability tensor is introduced to account for a permeability much higher along the fracture compared to the cross section. However, this method is restricted to fracture growth only in $x$ - and $y$ coordinate directions. Our phase-field approach allows us to treat general full permeability tensors. We emphasize in particular on an derivation of a $3 \mathrm{~d}$ lubrication equation for computing the fracture pressure based on mathematically consistent interface conditions. Moreover, it is well known that the lubrication equation degenerates at the crack tip [18]. Since we extend to a $3 \mathrm{~d}$ lubrication approximation, the degeneration applies to the whole fracture-reservoir interface. Our proposed treatment is akin to a cake-region [31], where we interpolate between the fracture and reservoir permeabilities.

The paper is organized as follows: In Section 2, we provide the notation and model formulation. Next, in Section 3, the discretization and the solution algorithm are defined and existence of a generalized fracture diffraction problem is established. The final Section 4 presents numerical tests. Specifically in two dimensions, we consider crack growth and multiple joining cracks. In three dimensions, we compute a propagating pennyshape crack in homogeneous media.

\section{The equations for pressure, elasticity, and phase-field}

In this section, we first derive reservoir and fracture equations based on mass continuity equations. We then discuss a $3 \mathrm{~d}$ lubrication equation for computing the fracture permeability and show the solvability of the coupled pressure problem. In the final subsection, the variational three-field problem (pressure, elasticity, phase-field) is formulated.

In the following, let $\Omega \in \mathbb{R}^{d}, d=2,3$ be a smooth open and bounded set. We assume that the crack $\mathcal{C} \in$ $\mathbb{R}^{d-1}$ is contained compactly in $\Omega$, i.e., it does not reach the boundary. In the approximation, the fracture is approximated by a volume term and $\mathcal{C}$ becomes $\Omega_{\mathrm{F}}$ and $\partial \mathcal{C}=\Gamma(t)$. (see left Figure 2). We assume Dirichlet 
boundaries conditions $\partial \Omega_{D}:=\partial \Omega$ and notice that $\Omega:=\Omega_{R}(t) \cup \Omega_{F}(t) \cup \Gamma(t)$, where the two subdomains are time-dependent and $\Omega_{R}=\Omega \backslash\left(\Omega_{F}(t) \cup \Gamma(t)\right)$ is the reservoir domain. Let $I:=(0, T)$ denote the time interval.
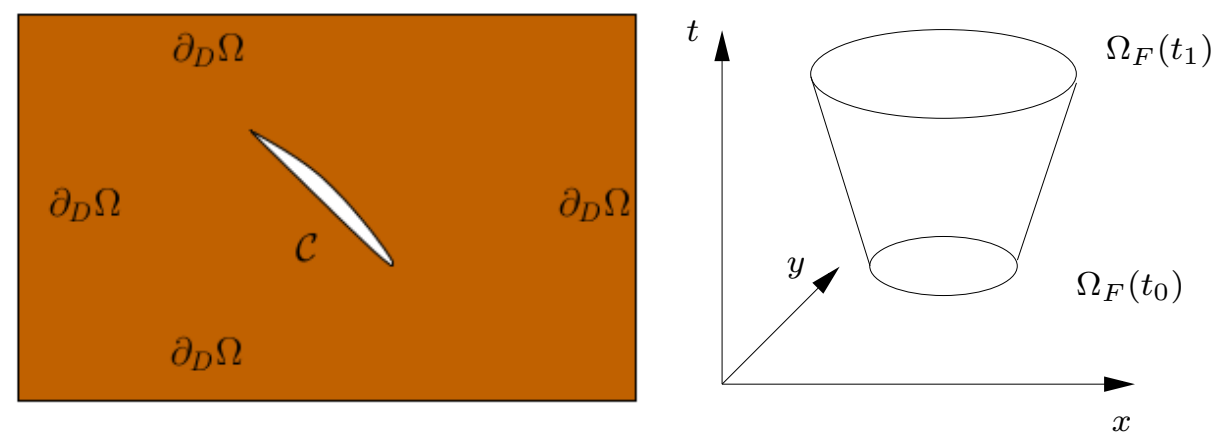

Figure 2: Illustration of the domain, boundary conditions and crack approximation at left. Space-time cylinder of the growing-in-time crack domain $\Omega_{F}(t)$ at right.

We first consider the mass continuity equations for fluid flow following $[12-15,21]$ and describe the two equations for the fracture and the reservoir, respectively:

$$
\begin{aligned}
\partial_{t} \rho_{F}+\nabla \cdot\left(\rho_{F} v_{F}\right) & =q_{F}-q_{L} \quad \text { in } \Omega_{F}(t) \times I, \\
\partial_{t}\left(\rho_{R} \phi_{R}\right)+\nabla \cdot\left(\rho_{R} v_{R}\right) & =q_{R} \quad \text { in } \Omega_{R}(t) \times I,
\end{aligned}
$$

where $q_{L}$ describes a leak-off term and $\phi_{R}$ is the reservoir fluid fraction. It should be noted that the porosity of the fracture is one. Here, the velocities are defined by Darcy's law (for the fracture and the reservoir, respectively):

$$
v_{j}=-\frac{K_{j}}{\eta_{j}}\left(\nabla p_{j}-\rho_{j} g\right)
$$

with $j=F, R$ (denoting the fracture or the reservoir). In the previous equations, $K_{j}$ is the permeability tensor, $\eta_{j}$ and $\rho_{j}$ are fluid's dynamic viscosity and its density, $g$ is the gravity and $q_{j}$ is a mass flow rate (usually $q_{j}$ is a point source modeling wells for fluid injection and production). A specific definition of Darcy's law in the fracture is derived in the next section.

The definition of the reservoir fluid fraction $\phi_{R}$ incorporates the volumetric strain $\varepsilon_{v}=\nabla \cdot u$ where $u$ denotes the vector-valued reservoir displacements and the gradient of the pressure:

$$
-\operatorname{div}(\sigma(u))+\alpha \nabla p=0 \quad \text { in } \Omega(t) \times I .
$$

Here $\alpha$ is Biot's coefficient, which is discussed in the next section, and the stress tensor of linear elasticity is defined by

$$
\sigma(u)=2 \mu e(u)+\lambda \operatorname{tr} e(u) I,
$$

where the Lamé coefficients are denoted by $\lambda>0$ and $\mu>0, e(u)=\frac{1}{2}\left(\nabla u+\nabla u^{T}\right)$, and $I$ is the identity in $d$-dimensions. 


\subsection{Reservoir pressure equation}

For the reservoir, we assume that the fluid is slightly compressible

$$
\rho_{R}=\rho_{R}^{0} \exp \left(c_{R}\left(p_{R}-p_{R}^{0}\right)\right) \approx \rho_{R}^{0}\left[1+c_{R}\left(p_{R}-p_{R}^{0}\right)\right],
$$

where $\rho_{R}^{0}$ denotes the (constant) reference density and $c_{R}$ is the reservoir fluid compressibility. Following $[12,14,15]$, the reservoir fluid fraction can be expressed as

$$
\phi_{R}=\phi_{R}^{0}+\frac{1}{M}\left(p_{R}-p_{R}^{0}\right)+\alpha\left(\nabla \cdot u-\varepsilon_{v}^{0}\right),
$$

with the initial porosity $0 \leq \phi_{R}^{0}$, the initial volumetric strain $\varepsilon_{v}^{0}$, the initial pressure $p_{R}^{0}$, the Biot-Willis constant (or Biot's coefficient) $0 \leq \alpha \leq 1$ with $\alpha \in[0,1]$. The Biot modulus is $M>0$.

As standard approximation in reservoir engineering, since $c_{R}$ is small, one uses $\rho_{R}=\rho_{R}^{0}$ in the mass and momentum (Darcy law) equations:

$$
\begin{aligned}
\partial_{t}\left(\rho_{R}^{0} \phi_{R}\right)+\nabla \cdot\left(\rho_{R}^{0} v_{R}\right) & =q_{R} \\
v_{R} & =-\frac{K_{R}}{\eta_{R}}\left(\nabla p_{R}-\rho_{R}^{0} g\right) .
\end{aligned}
$$

Taking in both equations $\rho_{R}=\rho_{R}^{0}$ and redefining $M$ due to the expansion (7), we write the mass conservation equation as:

$$
\rho_{R}^{0} \partial_{t}\left(\frac{1}{M} p_{R}+\alpha \nabla \cdot u\right)-\nabla \cdot \frac{K_{R} \rho_{R}^{0}}{\eta_{R}}\left(\nabla p_{R}-\rho_{R}^{0} g\right)=q_{R} \quad \text { in } \Omega_{R}(t) \times I .
$$

For later purposes and numerical stability (see for instance [21], p.57, or [34]), we further rewrite the fluid fraction as

$$
\phi_{R}=\phi_{R}^{0}+\left(\frac{3 \alpha^{2}}{3 \lambda+2 \mu}+\frac{1}{M}\right)\left(p_{R}-p_{R}^{0}\right)+\frac{3 \alpha}{3 \lambda+2 \mu}\left(\bar{\sigma}-\bar{\sigma}^{0}\right)
$$

where the mean stress is given by $\bar{\sigma}=\frac{3 \lambda+2 \mu}{3} \varepsilon_{v}-\alpha p_{R}$. Here, $c_{R}=\left(\frac{3 \alpha^{2}}{3 \lambda+2 \mu}+\frac{1}{M}\right)$. Thus, we obtain

$$
\rho_{R}^{0} \partial_{t}\left(\left(\frac{3 \alpha^{2}}{3 \lambda+2 \mu}+\frac{1}{M}\right) p_{R}+\frac{3 \alpha}{3 \lambda+2 \mu} \bar{\sigma}\right)+\nabla \cdot\left(\rho_{R}^{0} v_{R}\right)=q_{R} \quad \text { in } \Omega_{R}(t) \times I .
$$

Equation (9) is well balanced (between fluid and solid effects) if the time scale is chosen as Terzaghi's time $T_{R}$. The argument is based on a dimensionless form of Equation (9), i.e.,

$$
\frac{\rho_{R}^{0}}{T_{R}} \frac{P}{\Lambda}=\frac{\rho_{R}^{0}}{L} \frac{K_{R}}{\eta_{R}} \frac{P}{L}
$$

from which we obtain

$$
T_{R}=\frac{L^{2} \eta_{R}}{\Lambda K_{R}}
$$

Here, $P$ is the characteristic pressure, $L$ is the characteristic length (here the reservoir length), and $\Lambda$ is the characteristic size of $M$ and of the Lamé parameters $\mu$ and $\lambda$. We notice that $\mu, \lambda$ and $M$ are usually of similar order. 


\subsection{Fracture pressure equation}

For the fracture, we assume as before:

$$
\rho_{F}=\rho_{F}^{0} \exp \left(c_{F}\left(p_{F}-p_{F}^{0}\right)\right) \approx \rho_{F}^{0}\left[1+c_{F}\left(p_{F}-p_{F}^{0}\right)\right]
$$

where $\rho_{F}^{0}$ denotes the (constant) reference density. Again,

$$
\rho_{F}=\rho_{F}^{0},
$$

if the fracture fluid compressibility $c_{F}$ is assumed to be small.

In the following, we work with $\rho_{F}:=\rho_{F}^{0}$ in the term $\nabla \cdot\left(\rho_{F} v_{F}\right)$ to avoid nonlinear effects (for the same reason as before). The mass conservation in the fracture is then given by:

$$
\rho_{F}^{0} c_{F} \partial_{t} p_{F}+\nabla \cdot\left(\rho_{F}^{0} v_{F}\right)=q_{F} \quad \text { in } \Omega_{F}(t) \times I,
$$

i.e. (substituting the standard Darcy law),

$$
\rho_{F}^{0} c_{F} \partial_{t} p_{F}+\nabla \cdot \frac{K_{F} \rho_{F}^{0}}{\eta_{F}}\left(\nabla p_{F}-\rho_{F}^{0} g\right)=q_{F}-q_{L} \quad \text { in } \Omega_{F}(t) \times I .
$$

The unknown sink (often referred to as the leak-off) term $q_{L}$ in the near-tip and at the crack boundary contains the effects of their interaction with the surrounding poroelastic medium. In the Appendix, we provide a relationship of $q_{L}=\nabla \cdot\left(\rho_{F} v_{F}\right)$ based on the mass loss at the moving boundary Equation (11) is well balanced if Terzaghi's time in the fracture is chosen as

$$
T_{F}=\frac{L^{2} \eta_{F} c_{F}}{K_{F}}
$$

Since we have two Terzaghi time scales $T_{R}$ and $T_{F}$, the coupled reservoir-fracture model (9) and (11), is balanced if $T_{R} \approx T_{F}$. In this case, the only 'free' parameter would be the fracture compressibility $c_{F}$. However, if this parameter is known (which is frequently the case), then it yields $T_{F}<T_{R}$ because of the much higher permeability in the fracture than in the reservoir. Thus, the time step should be chosen according to $T_{F}$ in order to model the fracture dynamics. In fact, a realistic parameter choice (as taken in the numerical examples) indicates that $T_{F} \ll T_{R}$. Then, it yields $\phi_{R}=\phi_{R}^{0}+O\left(\frac{T_{F}}{T_{R}}\right)$ and $p_{R}=-M \alpha \operatorname{div} u+M \phi_{R}^{0}+O\left(\frac{T_{F}}{T_{R}}\right)$. At the leading order, the displacement $u \in \Omega_{R}$ is given by the Lamé-Navier-elasticity system with $\lambda$ being replaced by $\lambda+M \alpha^{2}$, i.e., $\sigma(u)=2 \mu e(u)+\left(\lambda+M \alpha^{2}\right) \operatorname{tr}(e) I$, with a given $\nabla p_{R}$ as right hand side force.

To account accurately for higher permeability along the fracture than to its cross section, we describe in the following a tensor-valued permeability. Moreover, this definition allows for arbitrary directions that is not restricted to one of the axis and is a generalization motivated by [27, 44]. The effective velocity for a given crack used in Equation (10) is computed as

$$
v_{F}=-\frac{K_{F}}{\eta_{F}}\left(\nabla p_{F}-\rho_{F} g\right)+v_{l e a k},
$$

where the leak-off velocity $v_{\text {leak }}$ is defined below. Here, the fracture permeability is a tensor that is defined in the following and derived in the Appendix. Its derivation is based on a $3 \mathrm{~d}$ extension of the standard $2 \mathrm{~d}$ lubrication equation, where we assume that the fracture is a piece-wise linear nonplanar fracture. 


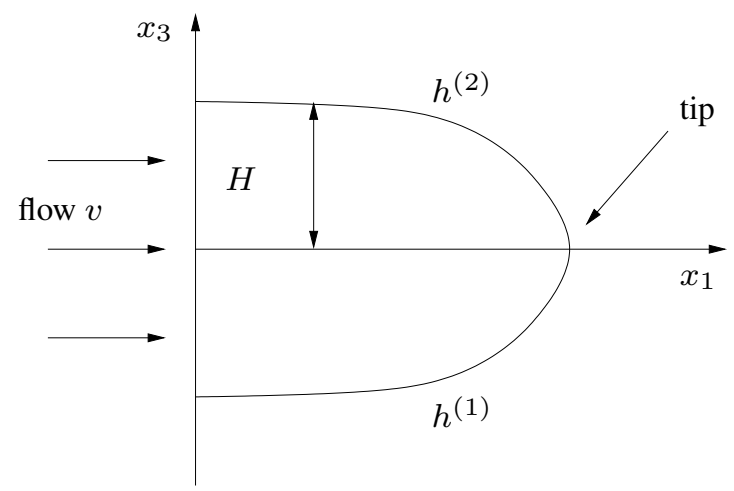

Figure 3: Sketch of the near-tip fracture region for the two-dimensional setting. Its boundary $x_{3} \pm h\left(x_{1}, x_{2}, t\right)$ moves in time. We assume that the characteristic width $H$ of the fracture is much bigger than the pore size of the porous medium. We recall that for $H=c_{0} l^{2 / 3}$ where $l$ is the pore size, a coupled model must be taken into account. In three dimensions, the $x_{2}$ coordinate goes into the plane and the one-dimensional curve $h\left(x_{1}, x_{2}, t\right)$ becomes a two-dimensional surface.

In three dimensions, we have

$$
K_{F}=\left[\begin{array}{ccc}
K & 0 & -K_{e} \partial_{x_{1}} \log \frac{K^{a b s}}{K_{a b}} \\
0 & K & -K_{e} \partial_{x_{2}} \log \frac{K^{a b s}}{K_{e}} \\
-K_{e} \partial_{x_{1}} \log \frac{K^{a b s}}{K_{e}} & -K_{e} \partial_{x_{2}} \log \frac{K^{a b s}}{K_{e}} & K
\end{array}\right]
$$

and

$$
v_{\text {leak }}=\left[\begin{array}{c}
0 \\
0 \\
\left(1-\frac{K_{e}}{K^{a b s}}\right)\left(\partial_{t} h^{(2)}+v_{\text {out }}^{(2)}\right)+\frac{K_{e}}{K^{a b s}}\left(\partial_{t} h^{(1)}+v_{\text {out }}^{(1)}\right)
\end{array}\right],
$$

where $K$ and $K_{e}$ are functions of $(x, t)$ given by (12) and (14). $K^{a b s}$ depends on $\left(x_{1}, x_{2}, t\right)$ and is given by (13). Specifically,

$$
\begin{aligned}
K\left(x_{3}\right) & =\frac{1}{2}\left(h^{(2)}\left(x_{1}, x_{2}, t\right)-x_{3}\right)\left(x_{3}-h^{(1)}\left(x_{1}, x_{2}, t\right)\right) \\
K^{a b s} & =\frac{\left(h^{(2)}\left(x_{1}, x_{2}, t\right)-h^{(1)}\left(x_{1}, x_{2}, t\right)\right)^{3}}{12} \\
K_{e}\left(x_{3}\right) & =\frac{\left(h^{(2)}\left(x_{1}, x_{2}, t\right)-x_{3}\right)^{2}}{12}\left(h^{(2)}\left(x_{1}, x_{2}, t\right)+2 x_{3}-3 h^{(1)}\left(x_{1}, x_{2}, t\right)\right) .
\end{aligned}
$$

Here, $h^{(1)}$ and $h^{(2)}$ are given functions that can be considered as negative and positive displacements of the crack, respectively. An illustration is provided in Figure 3. This means, $h^{(1)}+h^{(2)}=2 w$ where $w$ denotes the half-width of the fracture. The characteristic width $H$ of the fracture is chosen as $H:=\max \left\{-h^{(1)}, h^{(2)}\right\}$. Finally, the leak-off term is given by $D h^{(j)} / D t=-v_{\text {out }}^{(j)}$.

In two dimensions, the previous expressions reduce to

$$
K_{F}=\left[\begin{array}{cc}
K & -K_{e} \partial_{x_{1}} \log \frac{K^{a b s}}{K_{e}} \\
-K_{e} \partial_{x_{1}} \log \frac{K^{a b s}}{K_{e}} & K
\end{array}\right],
$$


and

$$
v_{\text {leak }}=\left[\begin{array}{c}
0 \\
\left(1-\frac{K_{e}}{K^{a b s}}\right)\left(\partial_{t} h^{(2)}+v_{\text {out }}^{(2)}\right)+\frac{K_{e}}{K^{a b s}}\left(\partial_{t} h^{(1)}+v_{\text {out }}^{(1)}\right)
\end{array}\right] .
$$

Specifically, the leak-off can be obtained from

$$
q_{L}=\operatorname{div}\left(\rho_{F} v_{\text {leak }}\right),
$$

where the leak-off velocity is (here in terms of the two-dimensional law)

$$
v_{\text {leak }}=\left[\begin{array}{c}
0 \\
\left(1-\frac{K_{e}}{K^{a b s}}\right)\left(\partial_{t} h^{(2)}+v_{\text {out }}^{(2)}\right)+\frac{K_{e}}{K^{a b s}}\left(\partial_{t} h^{(1)}+v_{\text {out }}^{(1)}\right)
\end{array}\right] .
$$

Thus,

$$
q_{L}=\partial_{x_{3}}\left(\rho_{F}\left(\left(1-\frac{K_{e}}{K^{a b s}}\right)\left(\partial_{t} h^{(2)}+v_{\text {out }}^{(2)}\right)+\frac{K_{e}}{K^{a b s}}\left(\partial_{t} h^{(1)}+v_{\text {out }}^{(1)}\right)\right)\right)
$$

Remark 2.1 The fracture width $2 w:=2 w(u)=h^{(1)}+h^{(2)}=-[u \cdot n]$ is the jump of the normal reservoir displacements and can be computed via its general definition

$$
2 w=-[u \cdot n]=-\left[u_{x} n_{x}+u_{y} n_{y}+u_{z} n_{z}\right],
$$

where $n$ is the unit normal on the crack surface.

Remark 2.2 Both two and three-dimensional laws are valid except at the interface between the fracture and the reservoir because of the degeneration of the terms $K^{\text {abs }}$. To avoid the singular behavior in modeling, computations and loss of regularity, we determine a new permeability-viscosity ratio $K_{\text {eff }}$ by interpolation between $K_{R} / \eta_{R}$ and $K_{F} / \eta_{F}$ in a so-called cake region [31] that is determined by the phase-field variable. Our definition of the cake region is $0<\varphi_{\text {low }} \leq \varphi \leq \varphi_{\text {high }}<1$. Specifically, outside the cake region, we use $K_{\text {eff }}=K_{R} / \eta_{R}$ with $\varphi>\varphi_{\text {high }}$ and $K_{\text {eff }}=K_{F} / \eta_{F}$ with $\varphi<\varphi_{\text {low }}$. The resulting interpolated permeability $K_{\text {eff }}$ is Lipschitz-continuous in time and space (see Proposition 2.2).

\subsection{A weighted pressure equation formulated as a diffraction problem}

In the following, we shall derive a single pressure equation in $\Omega:=\Omega_{F}(t) \cup \Omega_{R}(t)$ with the time-dependent interface $\Gamma(t):=\bar{\Omega}_{F}(t) \cap \bar{\Omega}_{R}(t)$ in which both sub-domain equations are described in the same dimension. This is in contrast to many other studies where a lower-dimensional fracture pressure equation is coupled to the reservoir pressure problem. In this work, the concept of phase-field modeling and therefore having an indicator function $\chi$ provides a uniform way to couple the two pressure equations in the same dimension.

To begin, we notice that the crack domain $\Omega_{F}(t)$ penetrates the poroelastic medium $\Omega_{R}(t)$ with the interface (i.e., the crack boundary) velocity $v_{I}$. In addition, crack-fluid and reservoir velocities are given by $v_{F}$ and $v_{R}$. An illustration is provided in Figure 4. In general, it appears reasonable to assume $v_{I} \cdot n \geq v_{F} \cdot n \geq v_{R} \cdot n$. In the next lines, we provide a mathematical compatible argument to cope with the interface velocity. The fluid mass conservation equations require consequently the interface-corrected continuity of fluxes (first principle Rankine-Hugoniot conditions):

$$
\rho_{R}^{0}\left(v_{\mathrm{R}}-v_{I}\right) \cdot n=\rho_{F}^{0}\left(v_{\mathrm{F}}-v_{I}\right) \cdot n \text {. }
$$


Mikelić, Wheeler, Wick

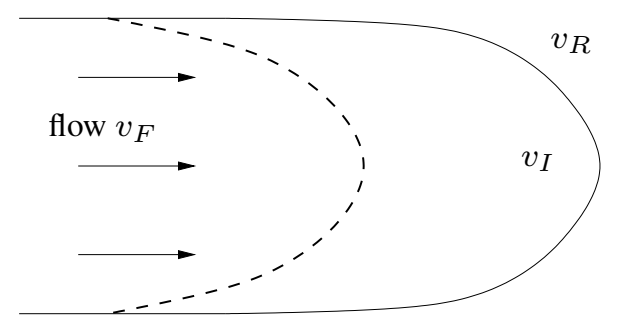

Figure 4: Illustration of the three velocities $v_{F}, v_{I}, v_{R}$.

The averaging property of the fluid mass conservation equations yields that any information of the tangential velocity is missing. For our proposed approximation for crack propagation, we assume $v_{I} n \neq 0$, and the continuity law

$$
v_{R} \cdot n=v_{I} \cdot n
$$

Combining these assumptions with (17) implies

$$
v_{F} \cdot n=v_{I} \cdot n=v_{R} \cdot n .
$$

Remark 2.3 This interface expression is compatible with $v_{I} \equiv 0$, which arises when the crack is fixed; that is $\Omega_{F}$ and $\Omega_{R}$ are time-independent and fixed. Then, (17) reduces to

$$
\rho_{R}^{0} v_{R} \cdot n=\rho_{F}^{0} v_{F} \cdot n .
$$

Remark 2.4 For detailed tip asymptotics of fluid-driven fracture, we refer the reader to [18, 19, 24, 39] and the many references cited therein.

We now turn our consideration to a single pressure formulation in $\Omega$. To this end, we introduce an indicator function $\chi$ to distinguish between the both sub-domains. We extend the previous equations on the whole domain $\Omega$ by defining $\chi_{\Omega_{R}}:=\chi_{\Omega_{R}}(t)=1$ in $\Omega_{R}(t)$ and $\chi_{\Omega_{F}}:=\chi_{\Omega_{F}}(t)=1$ in $\Omega_{F}(t)$, i.e., $\chi_{\Omega_{F}}=1-\chi_{\Omega_{R}}$. Thus, we have

$$
\begin{aligned}
& \chi_{\Omega_{R}}\left(\left(\frac{3 \alpha^{2}}{3 \lambda+2 \mu}+\frac{1}{M}\right)\right) \partial_{t} p_{R}+\chi_{\Omega_{R}} \nabla \cdot v_{R} \\
& \quad=\chi_{\Omega_{R}} \frac{q_{R}}{\rho_{R}^{0}}-\chi_{\Omega_{R}} \partial_{t}\left(\frac{3 \alpha}{3 \lambda+2 \mu} \bar{\sigma}\right) \quad \text { in } \Omega \times I, \\
& \chi_{\Omega_{F}} c_{F} \partial_{t} p_{F}+\chi_{\Omega_{F}} \nabla \cdot\left(v_{F}\right) \\
& \quad=\chi_{\Omega_{F}} \frac{q_{F}}{\rho_{F}^{0}} \quad \text { in } \Omega \times I .
\end{aligned}
$$

The resulting coupled equation is of generalized parabolic type with discontinuous coefficients and is a diffraction problem (see [32]). Specifically, the domain is divided into subdomains where two interface conditions must be satisfied. Namely, these are continuity of the solution variable $p$ and second, balance of forces.

The (generalized) pressure diffraction equation using Darcy's law (6) reads:

$$
\theta \partial_{t} p-\nabla \cdot K_{e f f}\left(\nabla p-\rho^{0} \tilde{g}\right)=\tilde{q} \quad \text { in } \Omega(t) \times I,
$$


where

$$
\begin{gathered}
\theta=\theta(x, t):=\chi_{\Omega_{R}} \theta_{R}+\chi_{\Omega_{F}} \theta_{F}=\chi_{\Omega_{R}}\left(\left(\frac{3 \alpha^{2}}{3 \lambda+2 \mu}+\frac{1}{M}\right)\right)+\chi_{\Omega_{F}} c_{F} \\
\tilde{q}=\tilde{q}(x, t):=\chi_{\Omega_{R}} \frac{q_{R}}{\rho_{R}^{0}}-\chi_{\Omega_{R}} \partial_{t}\left(\frac{3 \alpha}{3 \lambda+2 \mu} \bar{\sigma}\right)+\chi_{\Omega_{F}} \frac{q_{F}}{\rho_{F}^{0}} ; \quad \rho^{0}=\chi_{\Omega_{R}} \rho_{R}^{0}+\chi_{\Omega_{F}} \rho_{F}^{0}, \\
\tilde{g}=\chi_{\Omega_{R}} g+\chi_{\Omega_{F}}\left(g+K_{\text {eff }}^{-1} \frac{v_{\text {leak }}}{\rho_{F}^{0}}\right) .
\end{gathered}
$$

The interface conditions on $\Gamma(t):=\bar{\Omega}_{F}(t) \cap \bar{\Omega}_{R}(t)$ are given by

$$
\begin{aligned}
{[p] } & =0, \\
{\left[K_{e f f}\left(\nabla p-\rho^{0} \tilde{g}\right)\right] \cdot n } & =0,
\end{aligned}
$$

where $[\cdot]$ denotes the jump.

The existence and uniqueness of the pressure diffraction problem using the above interface and the initial conditions

$$
\left.p_{F}\right|_{t=0}=p_{F}^{0} \quad \text { in } \Omega_{F}(0) \quad \text { and }\left.\quad p_{R}\right|_{t=0}=p_{R}^{0} \quad \text { in } \Omega_{R}(0) .
$$

is established in the next section. We finish this section with the following remarks:

Remark 2.5 (Computation of the indicator function) The indicator function $\chi$ is computed via a phase-field function which is defined in Section 2.5.

Remark 2.6 (Lower-dimensional fracture pressure equations) Many research publications concentrate on the accurate description of the fluid equation in the fracture. Often, the fracture is identified as a lower dimensional manifold (a line in $2 d$ or a plane in $3 d$ ) on which the lubrication equation is used, for instance in [22, 33, 41]. Our strategy is different because the phase-field already requires a volume approximation and can be used as indicator function as previously described. Consequently, we avoid a four-field problem (reservoir pressure, crack lubrication pressure, elasticity, and phase-field) and corresponding development of specific approximations in lower-dimensional spaces.

\subsection{Solvability of the pressure diffraction interface problem}

In the present section, we discuss solvability and regularity of the pressure diffraction problem. The weak (variational or virtual work) formulation reads:

Problem 2.1 (Pressure diffraction problem on time-dependent domains) Find $p \in L^{\infty}\left(I ; H^{1}(\Omega)\right), \partial_{t} p \in$ $L^{2}(\Omega \times I)$ such that

$$
\int_{I} \int_{\Omega} \theta \partial_{t} p \psi d x d t+\int_{I} \int_{\Omega} K_{e f f}\left(\nabla p-\rho^{0} \tilde{g}\right) \nabla \psi d x d t=\int_{I} \int_{\Omega} \tilde{q} \psi d x d t
$$

for all $\psi \in L^{2}\left(I ; H^{1}(\Omega)\right)$; and

$$
\left.p\right|_{t=0}=p^{0} .
$$


Problems of type (22)-(23) have been treated in [32], page 232, for the case $g=0$. In this section, we extend these results because [32] relies on higher regularity of the coefficients.

In order to overcome this challenge, we use a standard approach from moving boundary problems and transform the current configuration at time $t$ back to its initial configuration at time $t=0$. This transformation can be realized by using the corresponding displacement field $u$ in $\Omega_{R}(t)$. Here, we suppose

$$
x=u(y, t)+y, t \in I, \frac{D y}{D x} \in W^{1, \infty}(I \times \Omega)^{9}, J(t)=\operatorname{det} \frac{D y}{D x}>0 \text { and } u \in C^{1}(\bar{I} \times \Omega)^{3} .
$$

With these assumptions, the interface $\Gamma(t)$ is $C^{1}$. Moreover, the normal vector $n$ and the velocity $v=\partial_{t} u$ are continuous. Then we have the following result

Proposition 2.2 Let $p^{0} \in H^{1}(\Omega), \tilde{q} \in L^{2}(\Omega \times I)$, v leak $\in C^{1}\left(\bar{I} ; L^{\infty}(\Omega)\right)$ and let $K_{\text {eff }}$ be Lipschitz-continuous in time and space. We furthermore assume (24). Then the problem (22)-(23) has a unique solution $p \in$ $L^{\infty}\left(I ; H^{1}(\Omega)\right) \cap H^{1}(I \times \Omega)$. Regularity in each of the two subdomains $\Omega_{F}$ and $\Omega_{R}$ depend on the smoothness of the interfaces and the initial datum.

Proof. We use the diffeomorphism $x=u(y, t)+y$. Its inverse transforms $\Gamma(t)$ to $\Gamma(0)$ and the part of $\Omega$ far from the interface is unchanged. The weak formulation (22) transforms to

$$
\begin{gathered}
\int_{I} \int_{\Omega} \theta^{0} \partial_{t} \tilde{p} \psi J(t) d y d t+\int_{I} \int_{\Omega} K_{e f f}^{0}\left(\frac{D y}{D x} \nabla_{y} \tilde{p}-\rho^{0} \tilde{g}\right) \frac{D y}{D x} \nabla_{y} \psi J(t) d y d t= \\
\int_{I} \int_{\Omega} q^{0} \psi J(t) d x d t, \quad \psi \in L^{2}\left(I ; H^{1}(\Omega)\right) .
\end{gathered}
$$

Here $\tilde{p}(y, t)=p(x(y, t), t)$, and $\theta^{0}$ (respectively $K_{\text {eff }}^{0}$ and $q^{0}$ ) is $\theta$ with $\chi_{a}(t), a=R, F$, replaced by $\chi_{a}(0)$. Letting $\psi=\partial_{t} \tilde{p}$, we have

$$
\begin{gathered}
\int_{\Omega} \theta^{0}\left(\partial_{t} \tilde{p}\right)^{2} J(t) d y+\int_{\Omega} K_{\text {eff }}^{0} \frac{D y}{D x} \nabla_{y} \tilde{p} \cdot \frac{D y}{D x} \partial_{t} \nabla_{y} \tilde{p} J(t) d y= \\
\int_{\Omega} q^{0} J(t) \partial_{t} \tilde{p} d x d t+\int_{\Omega} K_{\text {eff }}^{0} \rho^{0} \tilde{g} \cdot \frac{D y}{D x} \partial_{t} \nabla_{y} \tilde{p} J(t) d y .
\end{gathered}
$$

Next we use that $\rho^{0}$ does not depend on time and obtain

$$
\begin{aligned}
& \int_{\Omega} K_{e f f}^{0} \rho^{0} \tilde{g} \cdot \frac{D y}{D x} \partial_{t} \nabla_{y} \tilde{p} J(t) d y=\partial_{t} \int_{\Omega} K_{e f f}^{0} \rho^{0} \tilde{g} \cdot \frac{D y}{D x} \nabla_{y} \tilde{p} J(t) d y- \\
& \left.\int_{\Omega} K_{e f f}^{0} \rho^{0} \tilde{g} \cdot \partial_{t}\left(\frac{D y}{D x}\right) \frac{D y}{D x}{ }^{-1}\right) \frac{D y}{D x} \nabla_{y} \tilde{p} J(t) d y-\int_{\Omega} \partial_{t}\left(K_{e f f}^{0} \rho^{0} \tilde{g}\right) \cdot\left(\frac{D y}{D x} \partial_{t} \nabla_{y} \tilde{p} J(t) d y .\right.
\end{aligned}
$$

For the second term on the left hand side of (26) we have

$$
\begin{gathered}
\int_{\Omega} K_{\text {eff }}^{0} \frac{D y}{D x} \nabla_{y} \tilde{p} \cdot \frac{D y}{D x} \partial_{t} \nabla_{y} \tilde{p} J(t) d y=\frac{1}{2} \frac{d}{d t} \int_{\Omega} K_{\text {eff }}^{0} \frac{D y}{D x} \nabla_{y} \tilde{p} \cdot \frac{D y}{D x} \nabla_{y} \tilde{p} J(t) d y- \\
\int_{\Omega} \partial_{t}\left(J K_{\text {eff }}^{0}\right) \cdot \frac{D y}{D x} \nabla_{y} \tilde{p} J(t) d y-\int_{\Omega} K_{e f f}^{0} \frac{D y}{D x} \nabla_{y} \tilde{p} \cdot\left(\partial_{t}\left(\frac{D y}{D x}\right) \frac{D y}{D x}\right.
\end{gathered}
$$


Inserting (27)-(28) into (26) and using the hypothesis on the coefficients, we obtain

$$
\begin{aligned}
& \int_{0}^{t} \int_{\Omega}\left(\partial_{\tau} \tilde{p}\right)^{2} J(t) d y d \tau+\int_{\Omega}\left|\frac{D y}{D x} \nabla_{y} \tilde{p}(t)\right|^{2} J(t) d y \leq C\left(\int_{\Omega}\left|\frac{D y}{D x} \nabla_{y} \tilde{p}(0)\right|^{2} J(0) d y\right. \\
& \left.+\int_{0}^{t} \int_{\Omega}\left(q^{0}\right)^{2} J d y d \tau+\int_{0}^{t} \int_{\Omega}\left|\frac{D y}{D x} \nabla_{y} \tilde{p}(\tau)\right|^{2} J(\tau) d y d \tau+1\right) .
\end{aligned}
$$

Gronwall's inequality implies the a priori estimate

$$
\|\nabla p\|_{L^{\infty}\left(I ; H^{1}(\Omega)\right)}+\left\|\partial_{t} p\right\|_{L^{2}(\Omega \times I)} \leq C .
$$

Proving existence and uniqueness is now straightforward.

Remark 2.7 We finally emphasize that these theoretical considerations only hold for smooth cracks without branching and joining. Nevertheless, the associated computational method is still applicable to such settings, which is demonstrated in the numerical tests section. We finally recall that our crack permeability law is based on the assumption that the crack is piece-wise linear in the case of nonplanar cracks.

\subsection{The variational system of pressures, elasticity, and phase-field}

In this section, we recall the pressurized-crack phase-field modeling and discuss its coupling with the pressure diffraction equations. The concept of phase-field modeling in elasticity is based on energy minimization, which is described in detail in $[8,20]$. The extension and rigorous analysis including a fixed pressure is considered in $[35,36]$ and a corresponding numerical solution algorithm formulated in [46]. The mathematical classification of the three-field pressure-elasticity-phase-field problem is nonstandard. The Biot pressure equation is of generalized parabolic type that explicitly includes the time derivative on the pressure variable and the volumetric strain. The elasticity equation and the phase-field equation are both quasi-stationary. However, the latter one assumes a nonnegativity constraint on the time derivative. Consequently, analysis and implementation of this formulation are challenging and require careful design. Our approach is based on an extension of the Biot system, which is frequently used in subsurface modeling in a deformable porous medium. In fact, this system is a multiscale problem which can be identified on the micro-scale as fluid-solid interaction and details on the interface law are found in [38].

Let us denote by $(\cdot, \cdot)_{A},\|\cdot\|_{A}$ the usual $L^{2}$-inner product and norm on $A$. If $A=\Omega$ we drop the subscript for convenience. Let us denote $U:=L^{2}\left(0, T ; H^{1}(\Omega)\right), V:=H_{0}^{1}(\Omega)$ and $W:=L^{2}\left(0, T ; H^{1}(\Omega)\right)$ and let the previous time step solution $\left(p^{n-1}, u^{n-1}, \varphi^{n-1}\right) \in U \times V \times W$ be given. As derived in the previous sections, we work with the generalized pressure equation:

$$
\left(\theta \partial_{t} p, \phi\right)+\left(K_{e f f}\left(\nabla p-\rho^{0} \tilde{g}\right), \nabla \phi\right)-(\tilde{q}, \phi)=0 \quad \forall \phi \in U,
$$

and the elasticity equation with a pressure-force term in which we use $b=1$ and $\varphi_{+}:=\max \{0, \varphi\}$ (see [35]):

$$
\begin{aligned}
& \left(\left((1-\kappa) \varphi_{+}^{2}+\kappa\right) \mathcal{G} e(u), e(w)\right) \\
& \quad-(\alpha-1)\left(\varphi_{+}^{2} p, \operatorname{div} w\right)+\left(\varphi_{+}^{2} \nabla p, w\right)=0 \quad \forall w \in V,
\end{aligned}
$$

where $\kappa>0$ and $\varepsilon>0$ are the phase-field regularization parameters with their relationship $\kappa \ll \varepsilon$ [7] with $\kappa \approx 0$. The rank- 4 Gassmann tensor $\mathcal{G}$ for an isotropic poroelastic medium is given by

$$
\mathcal{G} e(u)=\sigma(u)=2 \mu e(u)+\lambda \operatorname{tr} e(u) I .
$$


Finally, we have the phase-field equation

$$
\begin{array}{r}
(1-\kappa)\left(\varphi_{+} \mathcal{G} e(u): e(u), \psi\right)-2(\alpha-1)\left(\varphi_{+} p \operatorname{div} u, \psi\right)+2\left(\varphi_{+} \nabla p u, \psi\right) \\
+G_{c}\left(-\frac{1}{\varepsilon}(1-\varphi, \psi)+\varepsilon(\nabla \varphi, \nabla \psi)\right)=0 \quad \forall \psi \in W,
\end{array}
$$

subject to the irreversibility constraint

$$
\partial_{t} \varphi \leq 0
$$

Here, $G_{c}$ stands for the critical energy release rate that is related to the fracture toughness.

In the previous derivation of the phase-field model, we follow [35, 36], and use the interface condition between the Biot system defined in $\Omega$ and a pressurized fracture on $C$, namely

$$
\sigma n=(\mathcal{G} e(u)-\alpha p I) n=-p_{f} n,
$$

and we obtain a phase-field formulation for crack modeling in a porous medium. The new variable denoted by $\varphi$ is the so-called phase-field function, which interpolates between the unbroken and the broken states of the material. In our previous studies [36, 46], the emphasis was on the verification of a phase-field approach for pressurized cracks with a given (fixed) pressure. Now, this framework is extended to propagating fluid-filled cracks in which Biot's pressure equation is coupled with the two-field elasticity phase-field framework, leading to a three-field problem.

In the following, we use the fact that we work in a quasi-static regime and use an incremental formulation. Thus, the time derivatives in the 1 st and the 3 rd equation are replaced by the backward difference quotient

$$
\begin{aligned}
& \partial_{t} p \approx \partial_{\Delta t} p=\frac{p-p^{n-1}}{\Delta t}, \\
& \partial_{t} u \approx \partial_{\Delta t} u=\frac{u-u^{n-1}}{\Delta t}, \\
& \partial_{t} \varphi \approx \partial_{\Delta t} \varphi=\frac{\varphi-\varphi^{n-1}}{\Delta t},
\end{aligned}
$$

where $\Delta t>0$ denotes the time step parameter and $p:=p^{n}, u:=u^{n}, \varphi:=\varphi^{n}$ the present solution and $p^{n-1}, u^{n-1}, \varphi^{n-1}$ the solution to the previous time step.

To solve the three-field problem, we employ the incremental formulation from now on and formulate bi-linear forms for the pressure and linear elasticity, and a semi-linear form for the nonlinear phase-field as follows:

$$
\begin{aligned}
A_{0}(p, \phi)= & \Delta t^{-1}\left(\theta\left(p-p^{n-1}\right), \phi\right) \\
& +\left(K_{e f f}\left(\nabla p-\rho^{0} \tilde{g}\right), \nabla \phi\right)-(\tilde{q}, \phi)=0 \quad \forall \phi \in U,
\end{aligned}
$$

and

$$
\begin{aligned}
A_{1}(u, w)= & \left(\left((1-\kappa) \varphi_{+}^{2}+\kappa\right) \mathcal{G} e(u), e(w)\right) \\
& -(\alpha-1)\left(\varphi_{+}^{2} p, \operatorname{div} w\right)+\left(\varphi_{+}^{2} \nabla p, w\right)=0 \quad \forall w \in V
\end{aligned}
$$


and

$$
\begin{aligned}
A_{2}(\varphi)(\psi)= & (1-\kappa)\left(\varphi_{+} \mathcal{G} e(u): e(u), \psi\right) \\
& -2(\alpha-1)\left(\varphi_{+} p \operatorname{div} u, \psi\right)+2\left(\varphi_{+} \nabla p u, \psi\right) \\
& +G_{c}\left(-\frac{1}{\varepsilon}(1-\varphi, \psi)+\varepsilon(\nabla \varphi, \nabla \psi)\right) \\
& +\Delta t^{-1}\left(\left(\Xi+\gamma\left(\varphi-\varphi^{n-1}\right)\right)_{+}, \psi\right)=0 \quad \forall \psi \in W .
\end{aligned}
$$

Here, $\gamma>0$ denotes the augmented Lagrangian penalization parameter and $\Xi \in L^{2}(\Omega)$ to treat the irreversibility constraint of crack growth [46]. The update routine for $\Xi$ is explained in Algorithm 1.

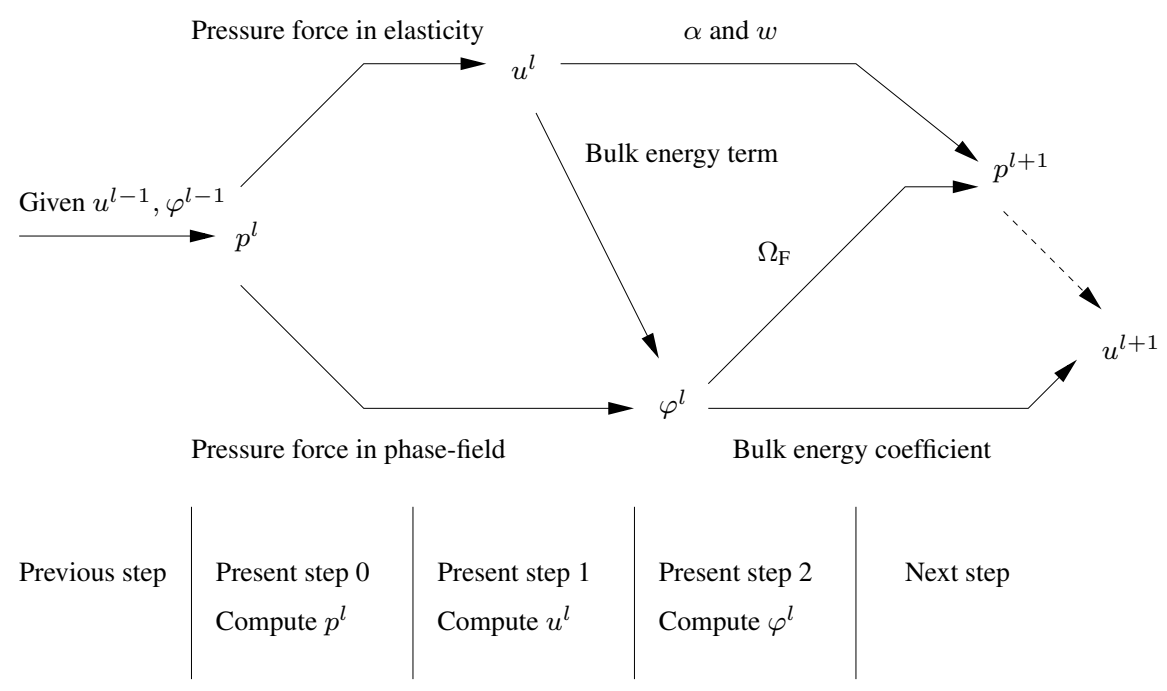

Figure 5: Explication how the three equations couple together. In each iteration step (within each time step), we compute first $p^{l}$, then $u^{l}$, and finally $\varphi^{l}$, where each variable influences the other two. Specifically, $\varphi^{l}$ alters the fracture domain $\Omega_{\mathrm{F}}$, which influences the computation of the new pressure $p^{l+1}$.

Coming back to the coupling of the single pressure $p$ with $u$ and $\varphi$, we deal with the situation as displayed in Figure 5. In each iteration step (within each time step), we compute first $p^{l}$, then $u^{l}$, and finally $\varphi^{l}$, where each variable influences the other two. 


\section{Discretization and Solution Algorithm}

\subsection{Galerkin finite element discretization}

The computational domain $\Omega$ is subdivided into quadrilateral or hexahedral element domains. The subproblems are discretized using $H^{1}$-conforming bilinear elements. Consequently, the discrete spaces have the property $U_{h} \subset U, V_{h} \subset V$ and $W_{h} \subset W$. In the following, we have five different indices $h, n$ denotes spatial and temporal steps, $m$ denoting the Newton steps, $l$ the inner loop coupling the equations, and $k$ used for the augmented Lagrangian loop. We use them where appropriate to avoid any confusion. Specifically, the Biot pressure problem and the elasticity problem are linear. The nonlinear phase-field problem is solved with Newton's method. For the iteration steps $m=0,1,2, \ldots$, it holds:

$$
A_{2}^{\prime}\left(\varphi^{h, m}\right)\left(\delta \varphi^{h}, \psi\right)=-A_{2}\left(\varphi^{h, m}\right)(\psi), \quad \varphi^{h, m+1}=\varphi^{h, m}+\omega \delta \varphi^{h}
$$

with a line search parameter $\omega \in(0,1]$. Here, we need the Jacobian of $A_{2}(\varphi)(\psi)$ applied to a direction $\delta \varphi$ :

$$
\begin{aligned}
A_{2}^{\prime}\left(\varphi^{h}\right)\left(\delta \varphi^{h}, \psi\right)= & (1-\kappa)\left(\delta \varphi^{h} \mathcal{G} e\left(u^{h}\right): e\left(u^{h}\right), \psi\right) \\
& -2(\alpha-1)\left(\delta \varphi^{h} p^{h} \operatorname{div} u^{h}, \psi\right)+2\left(\delta \varphi^{h} \nabla p^{h} w, \psi\right) \\
& +G_{c}\left(-\frac{1}{\varepsilon}\left(\delta \varphi^{h}, \psi\right)+\varepsilon\left(\nabla \delta \varphi^{h}, \nabla \psi\right)\right) \\
& +\gamma\left(\delta \varphi^{h}, \psi\right)_{\mathcal{B}(\varphi)}
\end{aligned}
$$

where

$$
\mathcal{B}\left(\varphi^{h}\right)=\left\{x \in(0, L)^{3} \mid \Xi^{h}+\gamma\left(\varphi^{h}-\varphi^{h, n-1}\right)>0\right\} .
$$

Summarizing, we deal with:

Problem 3.1 (Variational FE formulation for the pressure diffraction problem) Find $p^{h} \in U_{h}$ such that

$$
A_{0}\left(p^{h}, \phi\right)=0, \quad \forall \phi \in U_{h},
$$

and

Problem 3.2 (Variational FE formulation for linear elasticity) Find $u^{h} \in V_{h}$ such that

$$
A_{1}\left(u^{h}, w\right)=0, \quad \forall w \in V_{h},
$$

and

Problem 3.3 (Variational FE formulation for phase-field) Find $\varphi^{h} \in W_{h}$ such that

$$
A_{2}^{\prime}\left(\varphi^{h, m}\right)\left(\delta \varphi^{h}, \psi\right)=-A_{2}\left(\varphi^{h, m}\right)(\psi), \quad \varphi^{h, m+1}=\varphi^{h, m}+\omega \delta \varphi^{h},
$$

for all $\delta \varphi^{h} \in W_{h}$ and where $A_{2}$ and $A_{2}^{\prime}$ are given by (36) and (38).

In order to solve the linear subproblems, we use direct solvers (UMFPACK [16]) for the solution of Problem 3.1 and 3.3. The vector-valued displacement problem is solved with a PCG (preconditioned conjugate gradient) solver with SSOR (symmetric successive overrelaxation) preconditioning. 


\subsection{The fixed-stress algorithm}

In the fixed-stress splitting, we iterate the equations (34) and (35).

Problem 3.4 (Fixed-stress split) With the help of the given and fixed $\varphi$ from (36), i.e., the previous augmented Lagrangian step, let $\chi_{R}:=\left\{\varphi>\varphi_{\text {treas }}\right\}$ and $\chi_{F}:=\left\{\varphi \leq \varphi_{\text {treas }}\right\}$ be determined, respectively. For $l=$ $1,2,3, \ldots$, find $p^{l}$ such that

$$
\begin{aligned}
& \chi_{R}\left(\frac{1}{M}+\frac{\alpha^{2}}{3 \lambda+2 \mu}\right)\left(\frac{p^{l}-p^{n-1}}{\Delta t}, \phi\right)+\chi_{R}\left(K_{e f f}\left(\nabla p-\rho^{0} g\right), \nabla \phi\right) \\
& \quad-\chi_{R}(\tilde{q}, \phi)+\chi_{R} \alpha\left(\frac{\nabla \cdot u^{l-1}-\nabla \cdot u^{n-1}}{\Delta t}, \phi\right)-\chi_{R} \frac{\alpha^{2}}{3 \lambda+2 \mu}\left(\frac{p^{l-1}-p^{n-1}}{\Delta t}, \phi\right), \\
& \quad+\chi_{F} c_{F}\left(\frac{p^{l}-p^{n-1}}{\Delta t}, \phi\right)+\chi_{F}\left(K_{e f f}\left(\nabla p-\rho^{0} \tilde{g}\right), \nabla \phi\right)-\chi_{F}(\tilde{q}, \phi) \\
& =0 \quad \forall \phi \in U .
\end{aligned}
$$

Then, we solve for the displacements $u^{l}$ such that:

$$
\left(\left((1-\kappa) \varphi^{2}+\kappa\right) \mathcal{G} e\left(u^{l}\right), e(w)\right)=(\alpha-1)\left(\varphi^{2} p^{l}, \operatorname{div} w\right)-\left(\varphi^{2} \nabla p^{l}, w\right) \quad \forall w \in V .
$$

The iteration is completed if

$$
\max \left\{\left\|u^{l}-u^{l-1}\right\|,\left\|p^{l}-p^{l-1}\right\|\right\}<T O L_{F S} .
$$

\subsection{The solution algorithm}

Following [36], we use a quasi-static splitting approach for the fixed-stress-phase-field problem. Our strategy is described in Figure 5: at time step $t^{n}$, for the given iteration step $p^{l-1}, u^{l-1}, \varphi^{l-1}$, we solve first for $p^{l}$, then for $u^{l}$ and finally for the new $\varphi^{l}$. Thus,

Problem 3.5 (Augmented Lagrangian iteration in a time step $\left.t^{n}[46]\right)$ Let $\varphi^{h, l-1}$ be given from the previous iteration step. Find $p^{h, l} \in U_{h}$ and $u^{h, l} \in V_{h}$ with the fixed-stress iteration. Take $p^{h, l}$ and $u^{h, l}$ and solve finally for $\varphi^{h, l}$ :

$$
A_{2}^{\prime}\left(\varphi^{h, m, l}\right)\left(\delta \varphi^{h}, \psi\right)=-A_{2}\left(\varphi^{h, m, l}\right)(\psi), \quad \varphi^{h, m+1, l}=\varphi^{h, m, l}+\omega \delta \varphi^{h}
$$

for all $\delta \varphi^{h} \in W_{h}$ and where $A_{2}(\cdot)(\cdot)$ and $A_{2}^{\prime}(\cdot)(\cdot, \cdot)$ are given by (36) and (38).

The solution process is outlined in Algorithm 1. 
Mikelić, Wheeler, Wick

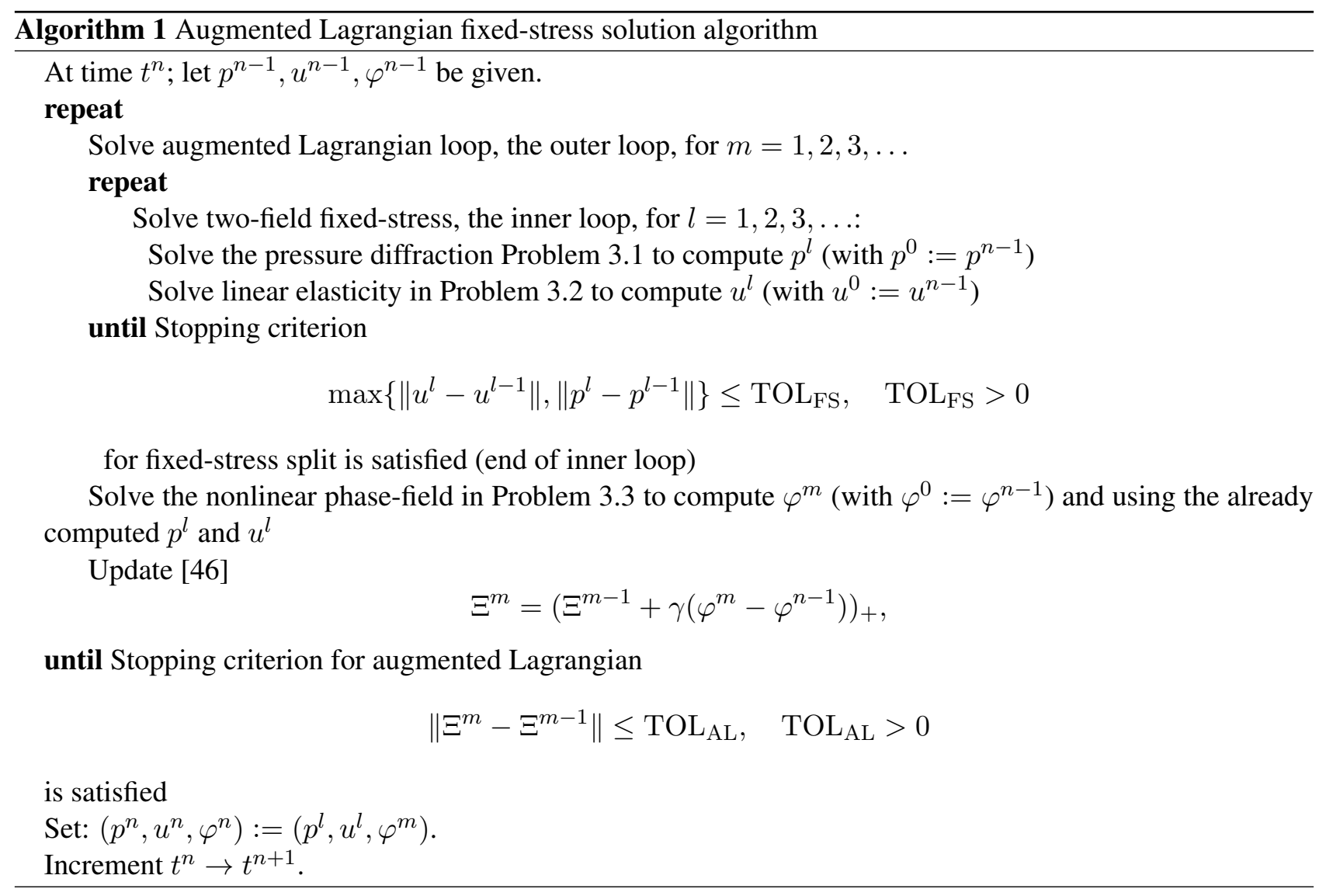




\section{Numerical Tests}

Several tests exploring the capabilities and attractive features of our method are presented in this final section. The goals of all tests are the observation of pressure development versus time (here we expect a pressure drop when the crack is growing); second, the fracture length as a function of time; and third, demonstration of nonaligned, nonplanar crack growth with joining of multiple fractures. Our proposed method applies to twoand three dimensional problems, and both are discussed in this section. In all examples, we compute the tests with $\alpha=0$ and $\alpha=1$ and each on three different grid levels to test the numerical stability of our approach. All examples are computed with the multiphysics template [47] in combination with deal.II's [1] step-31 for the usage of two different degree-of-freedom-handlers to build an iterative solution algorithm as needed for the fixed-stress splitting. In order to facilitate reading, we provide general information on all examples in the following.

\section{Geometry, grid and timestep parameters}

The geometry for all $2 \mathrm{~d}$ tests is displayed in Figure 6 and is $\Omega=(0,4)^{2}$. As boundary conditions we set the displacements zero on $\partial \Omega$. In $3 \mathrm{~d}$, in the cube $\Omega=(0,10)^{3}$, we prescribe a penny-shape crack with radius $r=1.0$ in the $y=5.0$-plane with mid-point $(5.0,5.0,5.0)$. The crack is approximated as a volume by extending it with the spatial discretization parameter $h$ in up- and downward $y$-direction, respectively. We compute 20 (2d) and 30 (3d) time steps with time step size $\Delta t=0.01$ (2d) and $\Delta t=0.005$ (3d), respectively. We note that the characteristic fracture time scale is

$$
T_{F}=\frac{L^{2} \eta_{F} c_{F}}{\tilde{K}_{F}}=\frac{1 \times 10^{-3} \times 10^{-8}}{10^{-8}}=10^{-3}
$$

in which we assumed a characteristic fracture length 1 and characteristic fracture permeability $\tilde{K}_{F}=10^{-4}$.

The initial mesh for the homogeneous $2 \mathrm{~d}$ tests is three times globally (see e.g. Figure 11 at left) and 4, 5, 6-times locally refined (see Figures 6 and 11). For the heterogeneous example in the introduction (see Figure 1), we refine the mesh seven times globally. In $3 \mathrm{~d}$, we use two times global and three and four times local refinement. In all cases, we already know the final crack pattern and refine locally a priori the crack region. A sophisticated method in which the mesh grows with the crack path is suggested in [28].

\section{Model parameters}

The augmented Lagrangian penalization parameter is $\gamma=10^{4}(2 \mathrm{~d})$ and $\gamma=10^{3}(3 \mathrm{~d})$. Several parameters and geometry-related issues depend on the spatial mesh size parameter $h$. Namely, for the regularization parameters we choose the relations $\kappa=10^{-6} \times h, \varepsilon=2 h_{\text {coarse }}=0.088$ (2d) and $\kappa=10^{-6} \times h, \varepsilon=2 h_{\text {coarse }}=1.09$ (3d). In addition, the initial (lower-dimensional) crack-line is extended by $h_{\text {coarse }}$ in normal direction to approximate it as a volume as discussed in [46].

\section{Pressure parameters}

In all examples, the gravity $g$ is set to zero and the fluid is only driven by the point source injection $q$. We inject fluid at a constant rate into the center of the domain,

$$
q:=\left\{\begin{array}{lll}
1 & \text { at }(2,2), \quad(\text { Example } 4.1), \\
1 & \text { at }(2,2),(1.45,1.55),(2.6,2.0), \\
1 & \text { at }(5,5,5), \quad(\text { Example } 4.3) .
\end{array} \quad\right. \text { (Example 4.2) }
$$

Furthermore, the permeability in the reservoir is $K_{R}=10^{-12}$. Next, $M=2.5 \times 10^{-8}, c_{F}=10^{-8}, \nu_{R}=$ $\nu_{F}=1.0 \times 10^{-3}, \rho_{R}^{0}=\rho_{F}^{0}=1$. Regarding the Biot coefficient, we perform computations with $\alpha=0$ and 
$\alpha=1$.

\section{Elasticity and phase-field parameters}

The fracture toughness is chosen as $G_{c}=1.0$. The mechanical parameters are $\mu=4.2 \times 10^{7}$ and $\lambda=2.8 \times 10^{7}$. The illustrative example in the introduction (see Figure 1) is computed with randomly varying Lamé parameters $\mu=4.2 \times 10^{6}-9.4 \times 10^{7}$ and $\lambda=2.6 \times 10^{6}-9.3 \times 10^{7}$.

\subsection{One crack in a homogeneous material ( $2 \mathrm{~d}$ case)}

The first set of tests is an extension to previous studies performed in $[6,46]$ in which the crack growth is due to a given pressure. Instead, we consider a well at position $(2,2)$ in which a fluid at a constant rate is injected. The initial crack has (total) length $l_{0}=0.4$ on $\Omega_{F}=(1.8,2.2) \times(2-h, 2-h) \subset \Omega$.

The first goal of this is to verify our algorithms for growing cracks due to an injected fluid into the crack. This is accomplished by studying the length of the fracture. Second, we observe the highest pressure versus time.

In Figure 8, the crack evolution at three different times is shown. In the Figures 9 and 10, the corresponding pressure field for both tests are provided. Our findings demonstrate the numerical stability since the pressure curve variation become smaller on finer meshes as monitored in Figure 7. Regarding the convergence of the length, we observe converging, but oscillating behavior in the $\alpha=1$ test case. We notice that the half-length evaluation is much more difficult than the pressure evaluation but it is also less important. As described in the previous sections, the pressure is characterized by different couplings to the other equations and in particular in the $\alpha=1$ case. Consequently, it is much more important to obtain numerical stability here. Furthermore, the pressure evolution curves represent physical behavior. As long as the crack does not move and we continue injecting a fluid, the pressure inside the fracture definitely increases. Once the fracture starts growing, the volume drastically increases and a pressure drop can be observed as displayed in Figure 7. A similar behavior is shown in Example 4.2 in Figure 13.

Finally, a higher fracture pressure is obtained with $\alpha=0$. For $\alpha=1$, div $u$ and $p$ are harmonic at the order $O\left(T_{F} / T_{R}\right)$ and $p=-M \operatorname{div} u+O\left(T_{F} / T_{R}\right)$, Figure 10 shows that $p$ achieves its minimum at the edges of the outer boundary (in blue) and its maximum around the crack surface.
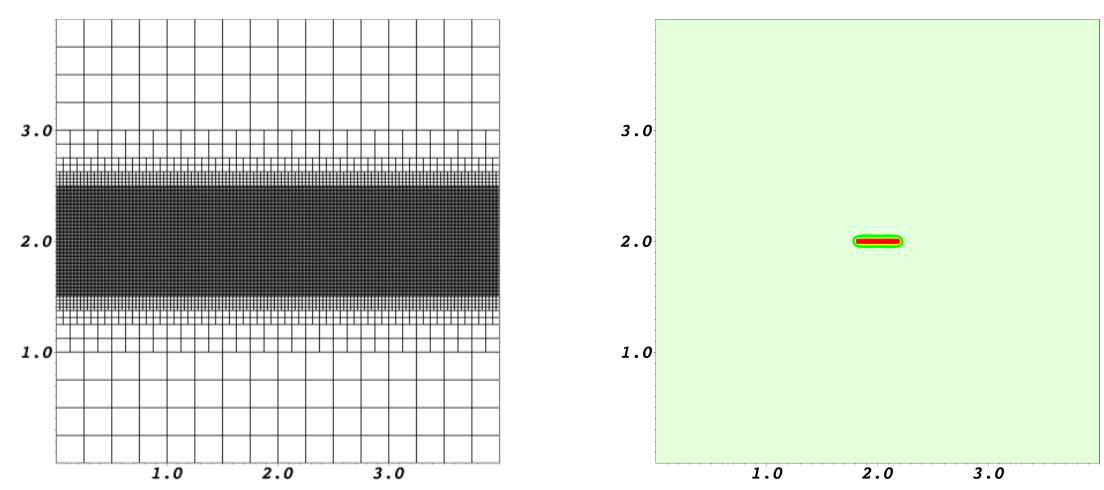

Figure 6: Example 1: Geometry using a locally prerefined mesh and initial crack. 

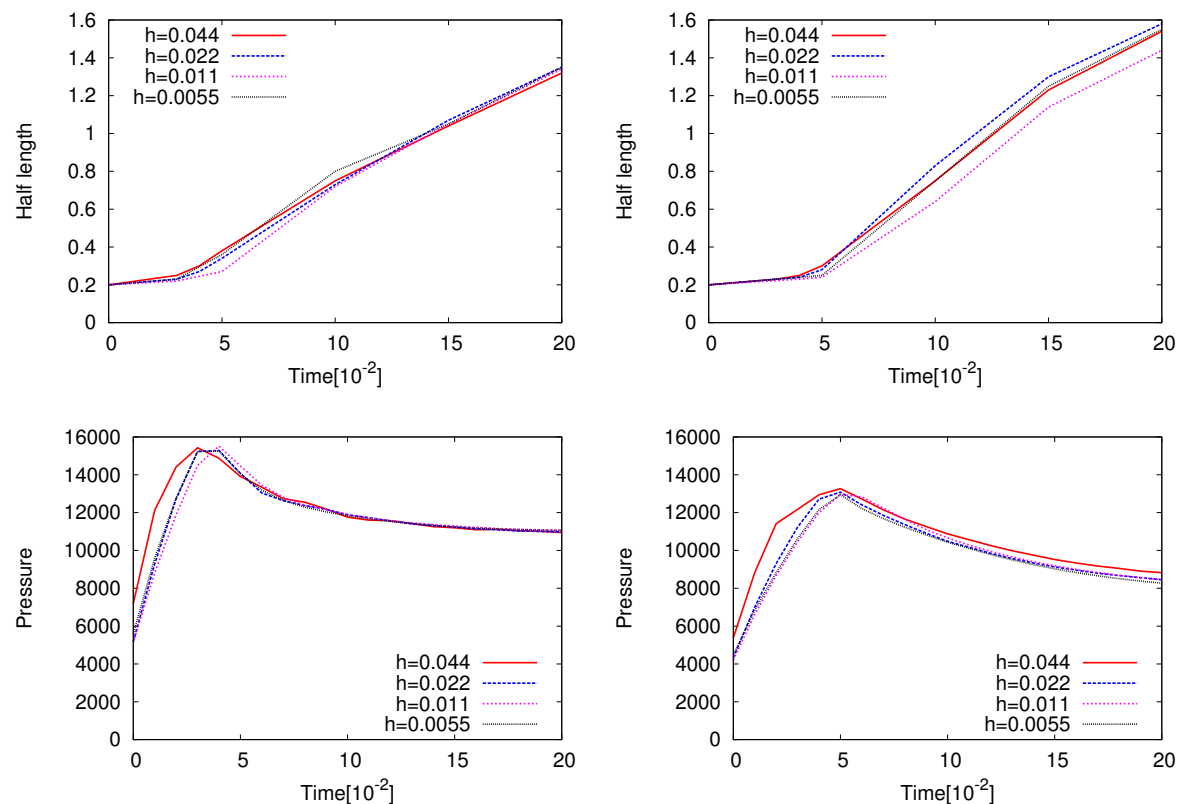

Figure 7: Example 1: Evolution of the half width (top) and of the fracture pressure (bottom) for the cases $\alpha=0$ (left) and $\alpha=1$ (right).
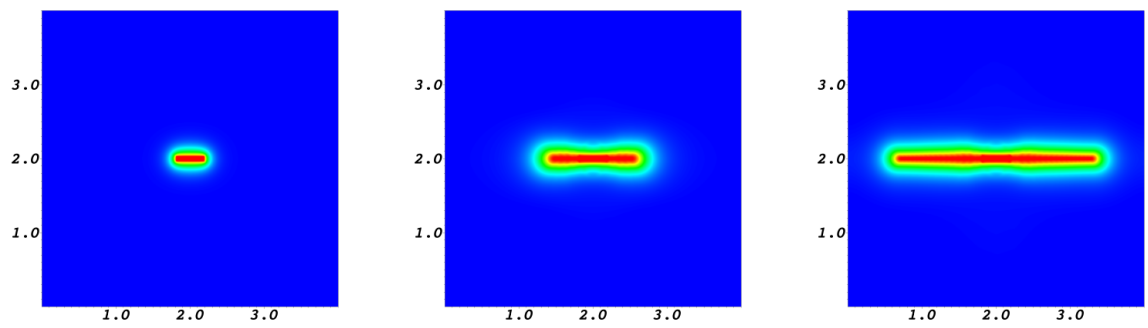

Figure 8: Example 1: Crack pattern in red color for $T=0,0.1,0.2$ for $\alpha=0$. The resulting crack pattern for $\alpha=1$ is similar.
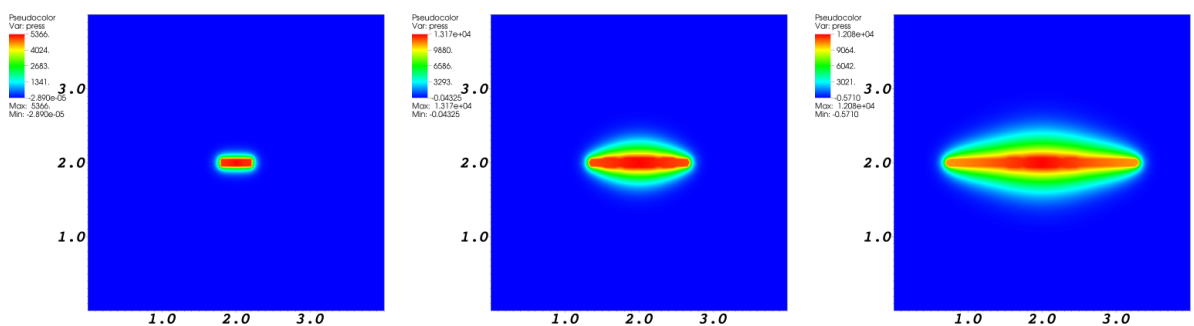

Figure 9: Example 1: Pressure distribution with $\alpha=0$ for $T=0,0.1,0.2$. 
Mikelić, Wheeler, Wick
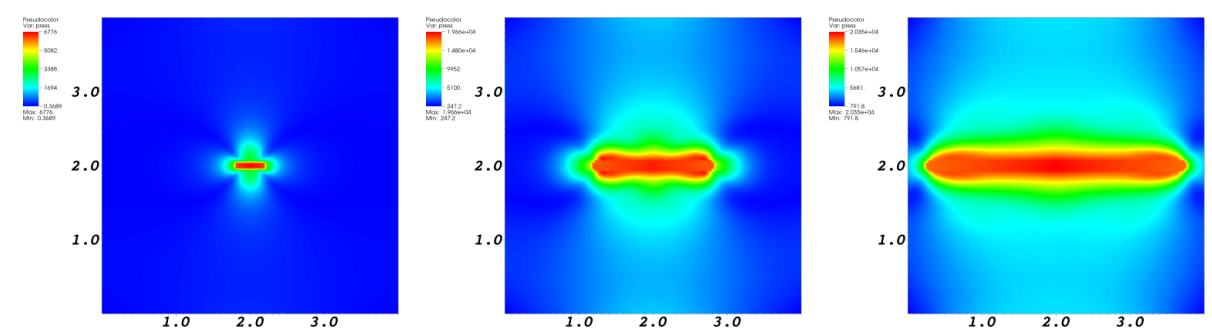

Figure 10: Example 1: Pressure distribution with $\alpha=1$ for $T=0,0.1,0.2$. 


\subsection{Three joining cracks in a homogeneous material ( $2 \mathrm{~d}$ case)}

In this example, we consider joining of three fractures. Apart from extending to multiple fractures, the configuration and all material parameters remain the same as in the previous numerical test. Two additional cracks are added. Here, crack 2 , is located at $(2.6-h, 2.6+h) \times(1.5,2.5) \in \Omega$. Finally, we add a third fracture that is not aligned with the axis and shows that our approach can deal with initial fractures of arbitrary orientation and location. The position of crack 3 is: $1.2 \leq x \leq 1.7, \quad x+3-h \leq y \leq x+3+h$. The goal of this second example is to study the pressure development versus time and the interaction between the three fractures.

As illustrated in Figure 11, we note that a sufficiently large region with a finer mesh is chosen a priori. All cracks never leave this fine mesh. Such a priori local mesh refinement already reduces the computational cost but is still not satisfactory. A promising approach that drastically reduces the computational cost has been recently applied to pressurized fractures with dynamics-in-time predictor-corrector mesh adaptivity [28]. In future studies, we intend to employ that approach for fluid-filled fractures.
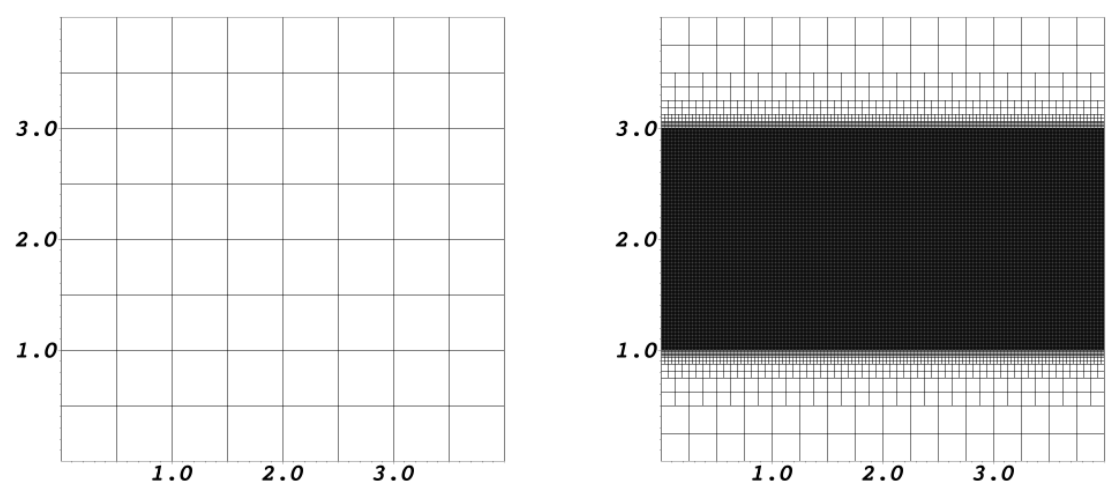

Figure 11: Example 2: Initial meshes: The domain $(0,4)^{2}$ is three times globally refined (left) and then 4, 5, 6 a priori locally refined. At right the finest mesh with $3+6$-times refinement is shown.

We observe in the Figures 12 the crack pattern for $\alpha=0$. We note that the crack profile for $\alpha=1$ is similar. In the Figures 14 and 15, the pressure profiles for $\alpha=0$ and $\alpha=1$ are displayed. In Figure 13, the pressure evolution for both test cases is shown. Here, we observe a higher fracture pressure for the decoupled case $\alpha=0$. As in the previous example, our findings demonstrate the numerical stability since the pressure curve variation becomes smaller on finer meshes.

As in the previous example, we analyze in more detail the pressure evolution. Here, we compare situations with $\alpha=0$ and $\alpha=1$ that show some similarities but also important differences; and both can be explained from physical observations. Using $\alpha=0$, geomechanics and fluid pressure are decoupled and consequently, displacement changes can not account to absorb fluid pressure, which leads to a higher overall pressure (see Figure 13 at left) than in the $\alpha=1$ test case. Both test cases show a pressure drop when the cracks are joining and suddenly fluid is allowed to move in a huge volume. However using $\alpha=0$, due to the three injection points the pressure still increases even under crack evolution. This is different in the $\alpha=1$ test case since here, the pressure equations recognize the displacement changes (due to the coupling) and the overall pressure does not increase under crack propagation. 
Mikelić, Wheeler, Wick
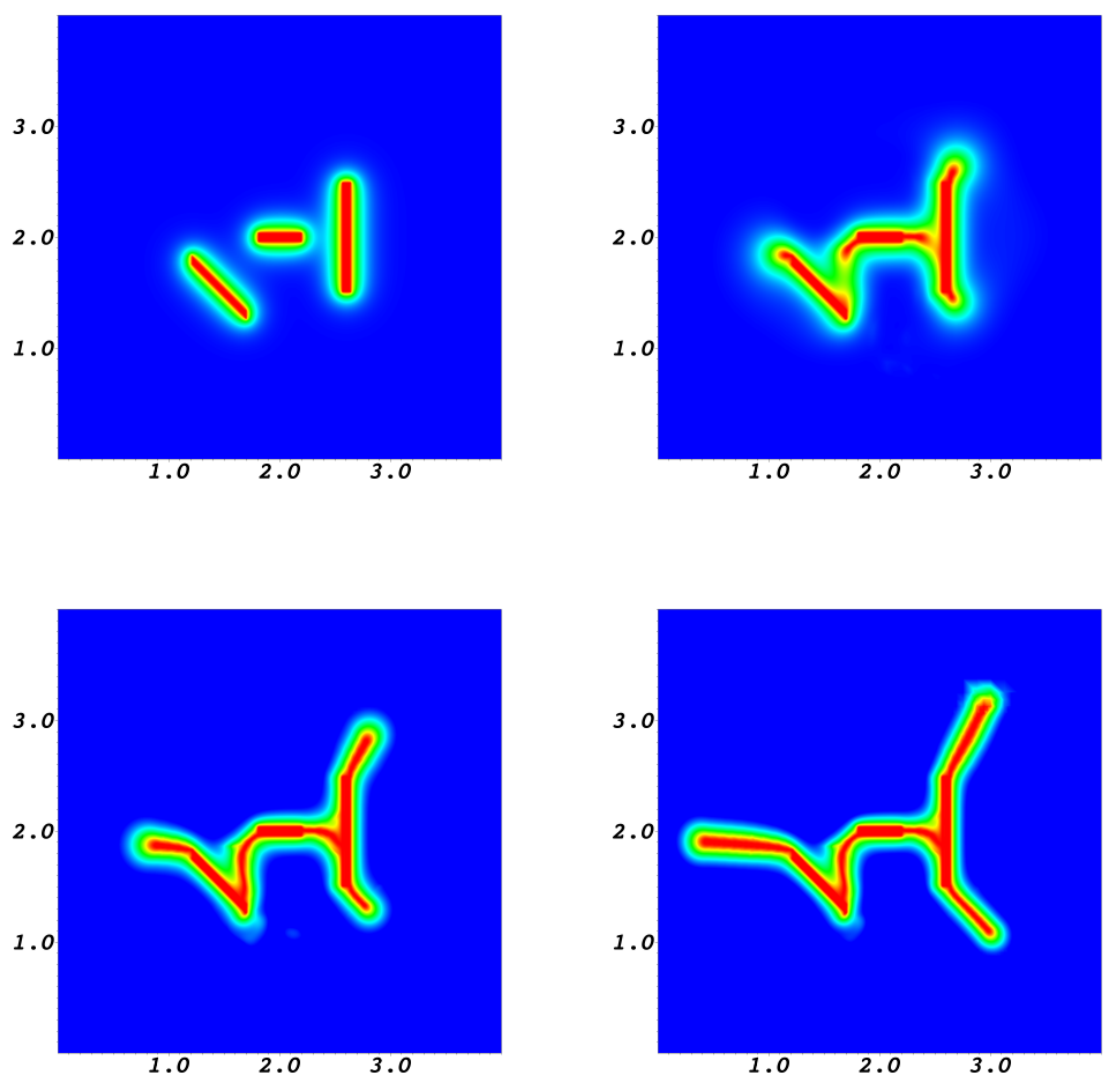

Figure 12: Example 2: Crack patterns are presented in red color for $T=0,0.7,0.15,0.2$ for $\alpha=0$. The crack pattern for $\alpha=1$ is similar.
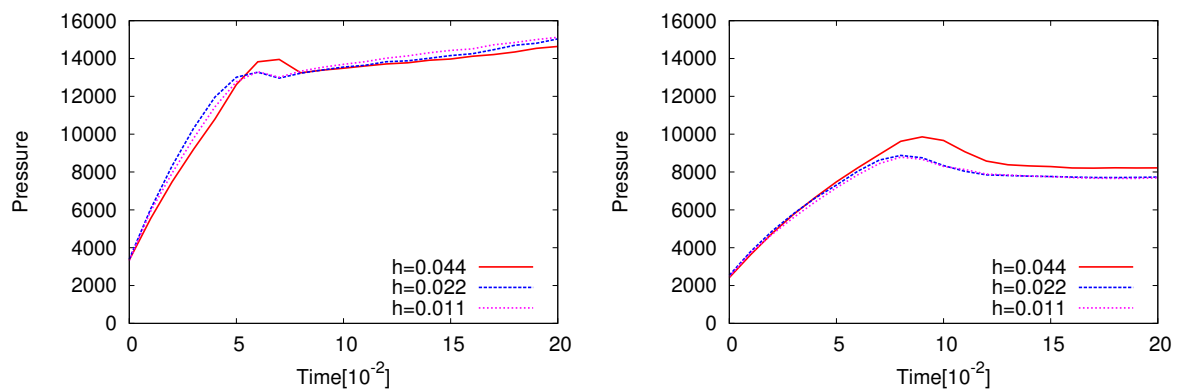

Figure 13: Example 2: Evolution of the fracture pressure with respect to time on different mesh levels. We observe a pressure drop when crack 1 joins the other two fractures. The fracture pressure is higher for the case $\alpha=0$ than for $\alpha=1$. 
Fluid-filled fractures in heterogeneous porous media
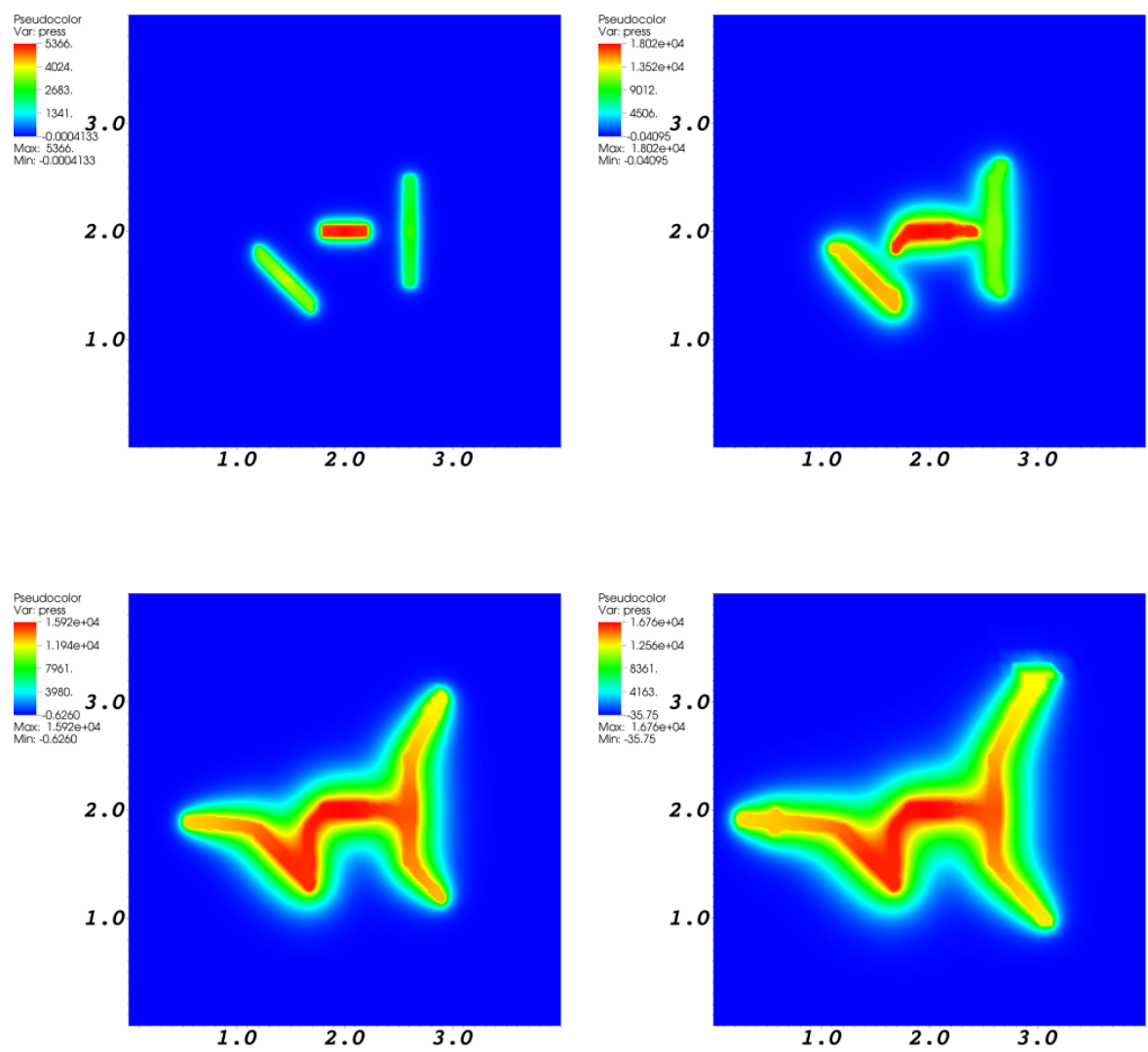

Figure 14: Example 2: We present the pressure distribution for $\alpha=0$ at $T=0,0.7,0.15,0.2$. 

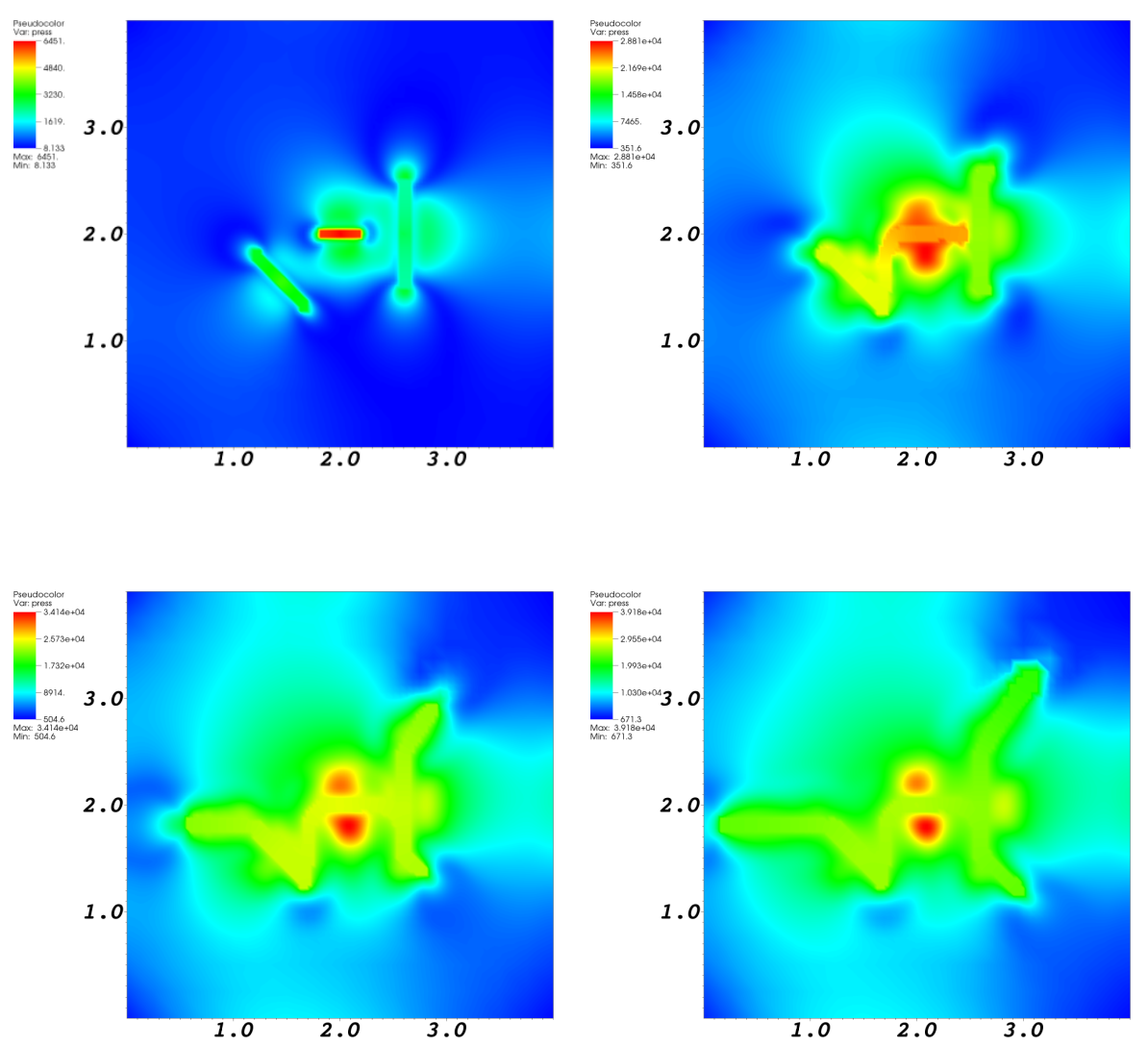

Figure 15: Example 2: We present the pressure distribution for $\alpha=1$ at $T=0,0.7,0.15,0.2$.

\subsection{A propagating penny-shape crack in 3d}

In this final example, we consider a propagating penny-shape crack in three dimensions. The locally-refined grid in $\Omega=(0,10)^{3}$ with hanging nodes is shown in Figure 16. The goals are to observe the pressure and the radius with respect to time. An extension of this setting to heterogeneous materials is discussed in [48].

Our findings show similar pressure behavior on two subsequent grid levels (see Figure 17). In addition, the fracture pressures for $\alpha=0$ and $\alpha=1$ are of similar order. The radius evolution with respect to time is displayed in the Figures 17. The crack and pressure patterns are provided in the Figures 18 and 19. 


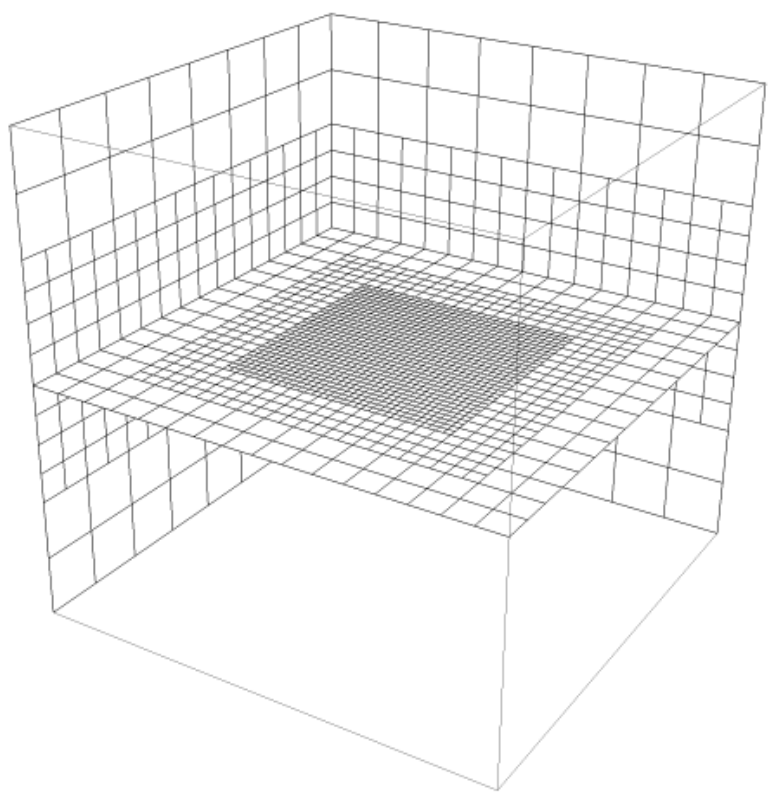

Figure 16: Example 3: Locally refined grid in the cube $\Omega=(0,10)^{3}$.
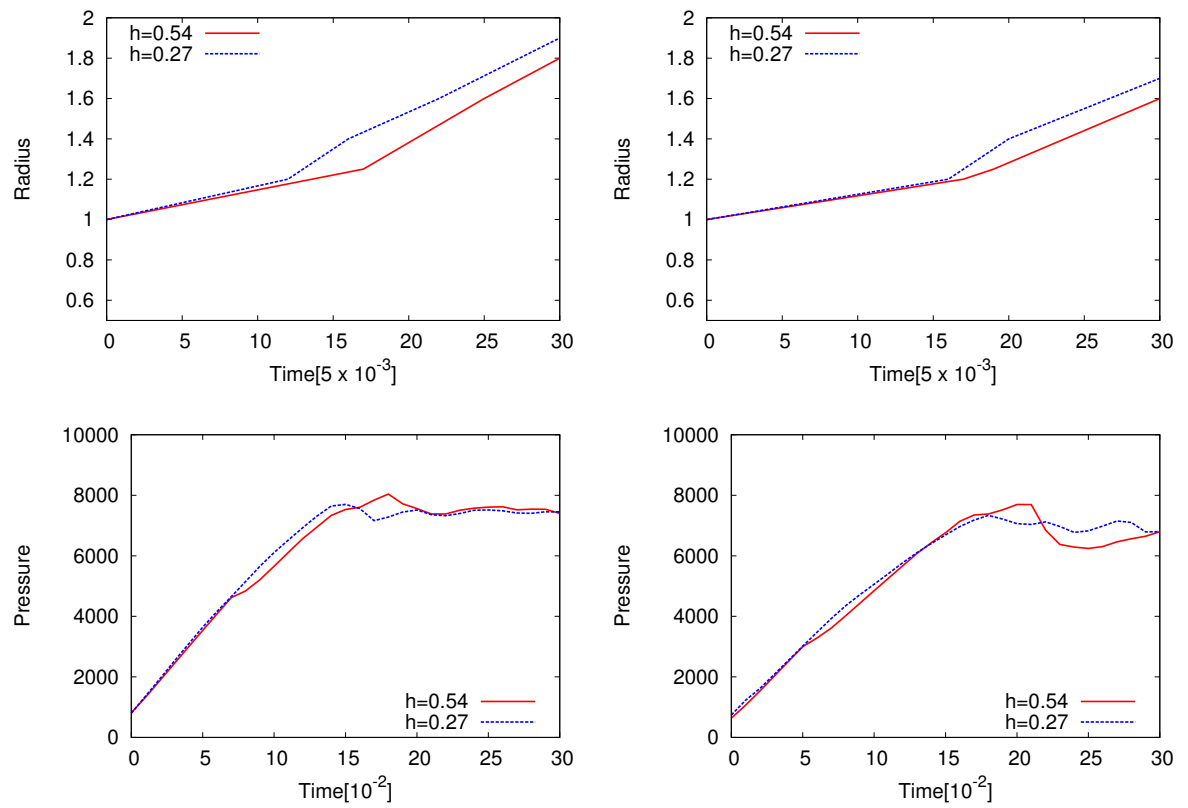

Figure 17: Example 3: radius of the penny-shape crack (top) and pressure (bottom) evolution for the cases $\alpha=0$ (left) and $\alpha=1$ (right). 
Mikelić, Wheeler, Wick
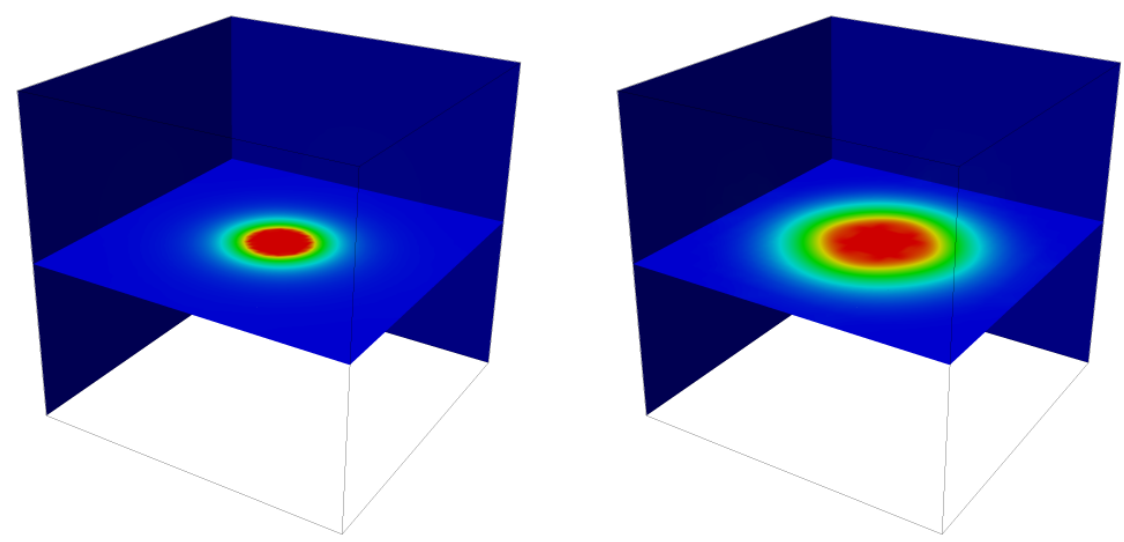

Figure 18: Example $3(\alpha=0)$ : Penny-shape crack pattern in red color at $T=0$ and final time $T=0.15$. The case $\alpha=1$ results in a similar crack pattern.
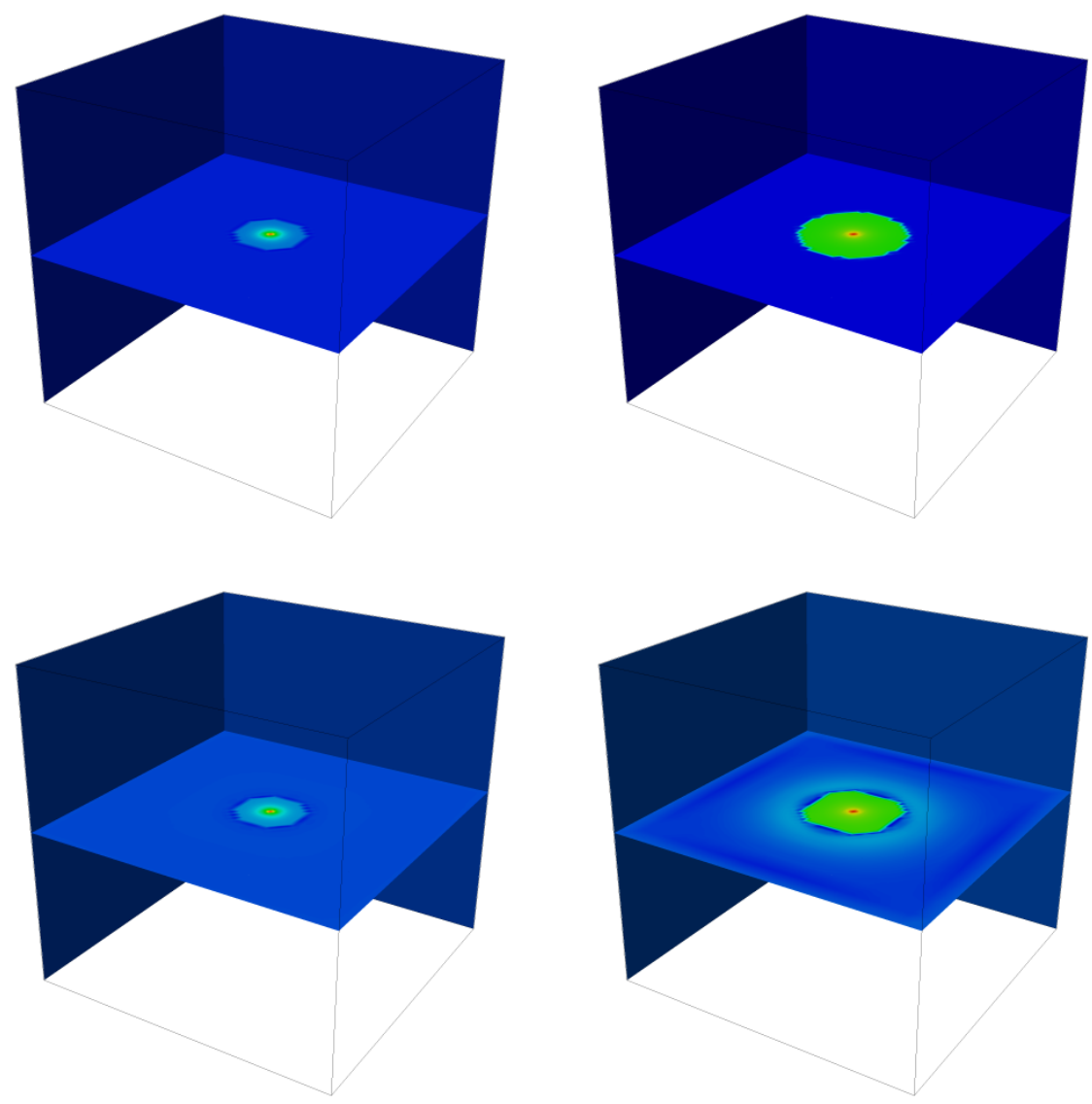

Figure 19: Example 3: case $\alpha=0$ (top) and case $\alpha=1$ (bottom): Pressure distribution at initial time $T=0$ (left) and final time $T=0.15$ (right). 


\section{Conclusions}

In this paper, we considered a phase-field formulation for fluid-filled crack propagation in porous media. The novelty is a coupling of Darcy pressure equations in a reservoir zone with fracture flow using an indicator variable. This function is obtained by solving a phase-field problem for the crack location. Due to the crack propagation, both the reservoir and fracture domains change in time and are treated as a moving boundary problem for which we proved existence and uniqueness. Specifically, based on a three-dimensional lubrication equation approximation, we derived a general expression for the fracture permeability. In addition to these theoretical results, we provided algorithmic details based on the fixed-stress splitting for solving the coupled fluid-solid interaction problem. Our theoretical developments are substantiated with numerical tests in two- and three dimensions and our findings show that our algorithms are robust and numerically stable.

\section{Appendix: Darcy's law for a flat 3D penny shape fracture}

In this section we give a short derivation of the relation between the velocity and the pressure gradient for the flow in a fracture. A flat $3 \mathrm{D}$ fracture $\Omega_{F}$ is characterized by the ratio $\varepsilon$ between its characteristic width $H$ in the direction $x_{3}$ and its characteristic horizontal length $L$ in directions $x_{1}$ and $x_{2}$; a sketch is provided in Figure 3 .

Here we assume the crack boundary propagates in a deformable porous medium and is given by

$$
x_{3}=h^{(2)}\left(x_{1}, x_{2}, t\right) \quad \text { for } x_{3} \geq 0 \quad \text { and } \quad x_{3}=h^{(1)}\left(x_{1}, x_{2}, t\right) \text { for } x_{3}<0 .
$$

Here $h^{(2)}$ and $h^{(1)}$ meet at $x_{3}=0$, the tip region. The fluid flow in the fracture is given by the incompressible Navier-Stokes equations

$$
\begin{gathered}
\frac{\partial v}{\partial t}+(v \cdot \nabla) v+\frac{1}{\rho_{F}} \nabla p-\nu_{F} \Delta v=0 \quad \text { in } \Omega_{F} \\
\operatorname{div} v=0 \quad \text { in } \Omega_{F}
\end{gathered}
$$

where $\rho_{F}$ is the fluid density and $\nu_{F}=\eta_{F} / \rho_{F}$ is the kinematic viscosity. Next, we derive a dimensionless form of the lubrication equation following the classical lubrication theory references (see e.g. [43]). Let $U$ be the characteristic velocity in directions $x_{1}$ and $x_{2}$ and $\mathbf{R e}=\frac{U L}{\nu_{F}}$ the Reynolds number. Then we set

$$
\begin{gathered}
\bar{t}=\frac{U}{L} t ; \quad x=\frac{x_{1}}{L}, \quad y=\frac{x_{2}}{L}, \quad z=\frac{x_{3}}{H}, \quad v_{x}=\frac{v_{1}}{U}, \quad v_{y}=\frac{v_{2}}{U}, \quad v_{z}=\frac{v_{3} L}{U H}, \\
\bar{p}=\frac{H^{2}}{\eta_{F} L U} p, \quad w^{(j)}(x, y, \bar{t})=\frac{1}{H} h^{(j)}\left(x_{1}, x_{2}, t\right) \quad \text { and } \quad \varepsilon=\frac{H}{L} .
\end{gathered}
$$

For simplicity, we skip the bars and denote the rescaled $\Omega_{F}$ with the same symbol. The system (42)-(43) becomes

$$
\begin{gathered}
\operatorname{Re} \varepsilon^{2}\left(\partial_{t} v_{x}^{\varepsilon}+\left(\left(v_{x}^{\varepsilon}, v_{y}^{\varepsilon}, v_{z}^{\varepsilon}\right) \cdot \nabla_{x, y, z}\right) v_{x}^{\varepsilon}\right)=-\partial_{x} p^{\varepsilon}+\varepsilon^{2} \Delta_{x, y} v_{x}^{\varepsilon}+\partial_{z z} v_{x}^{\varepsilon} \quad \text { in } \Omega_{F}, \\
\operatorname{Re} \varepsilon^{2}\left(\partial_{t} v_{y}^{\varepsilon}+\left(\left(v_{x}^{\varepsilon}, v_{y}^{\varepsilon}, v_{z}^{\varepsilon}\right) \cdot \nabla_{x, y, z}\right) v_{y}^{\varepsilon}\right)=-\partial_{y} p^{\varepsilon}+\varepsilon^{2} \Delta_{x, y} v_{y}^{\varepsilon}+\partial_{z z} v_{y}^{\varepsilon} \quad \text { in } \Omega_{F}, \\
\operatorname{Re} \varepsilon^{4}\left(\partial_{t} v_{z}^{\varepsilon}+\left(\left(v_{x}^{\varepsilon}, v_{y}^{\varepsilon}, v_{z}^{\varepsilon}\right) \cdot \nabla_{x, y, z}\right) v_{z}^{\varepsilon}\right)=-\partial_{z} p^{\varepsilon}+\varepsilon^{4} \Delta_{x, y} v_{z}^{\varepsilon}+\varepsilon^{2} \partial_{z z} v_{z}^{\varepsilon} \quad \text { in } \Omega_{F}, \\
\partial_{x} v_{x}^{\varepsilon}+\partial_{y} v_{y}^{\varepsilon}+\partial_{z} v_{z}^{\varepsilon}=0 \quad \text { in } \Omega_{F} .
\end{gathered}
$$

We suppose $\operatorname{Re} \varepsilon^{2} \ll 1$ and neglect the inertia term at the main order. We recall that the unit normal $n$ and 
tangential vectors $\tau^{(j)}, j=1,2$ can be expressed in the form

$$
n=\frac{-\varepsilon \partial_{x} w e^{1}-\varepsilon \partial_{y} w e^{2}+e^{3}}{\sqrt{1+\varepsilon^{2}\left|\nabla_{x, y} w\right|^{2}}} ; \quad \tau^{(1)}=\frac{e^{1}+\varepsilon \partial_{x} w e^{3}}{\sqrt{1+\varepsilon^{2}\left|\partial_{x} w\right|^{2}}} ; \quad \tau^{(2)}=\frac{e^{2}+\varepsilon \partial_{y} w e^{3}}{\sqrt{1+\varepsilon^{2}\left|\partial_{y} w\right|^{2}}} .
$$

Then for $v=U\left(v_{x}^{\varepsilon}, v_{y}^{\varepsilon}, v_{z}^{\varepsilon}\right)$ we have

$$
\begin{gathered}
v \cdot n=\frac{-U \varepsilon \partial_{x} w v_{x}^{\varepsilon}-U \varepsilon \partial_{y} w v_{y}^{\varepsilon}+U \varepsilon v_{z}^{\varepsilon}}{\sqrt{1+\varepsilon^{2}\left|\nabla_{x, y} w\right|^{2}}}=U \varepsilon\left(v_{z}^{\varepsilon}-\nabla_{x, y} w \cdot\left(v_{x}^{\varepsilon}, v_{y}^{\varepsilon}\right)\right)+O\left(\varepsilon^{3}\right) \\
v \cdot \tau^{(1)}=\frac{U v_{x}^{\varepsilon}+U \varepsilon^{2} \partial_{x} w v_{z}^{\varepsilon}}{\sqrt{1+\varepsilon^{2}\left|\partial_{x} w\right|^{2}}}=U v_{x}^{\varepsilon}+U \varepsilon^{2}\left(\partial_{x} w v_{z}^{\varepsilon}-\frac{v_{x}^{\varepsilon}}{2}\left(\partial_{x} w\right)^{2}\right)+O\left(\varepsilon^{4}\right)
\end{gathered}
$$

and analogously for $v \cdot \tau^{(2)}$. The velocity in the fracture is larger than in the surrounding porous medium. Hence we have at $z=w(x, y, t)$ :

$$
0=v \cdot \tau^{(1)}=U v_{x}^{\varepsilon}+U \varepsilon^{2}\left(\partial_{x} w v_{z}^{\varepsilon}-\frac{v_{x}^{\varepsilon}}{2}\left(\partial_{x} w\right)^{2}\right)+O\left(\varepsilon^{4}\right)
$$

and an analogous expression for $v \cdot \tau^{(2)}$. In the normal direction we have to impose the kinematic boundary condition: $\frac{D h}{D t}=-v_{\text {out }}$. The term $v_{\text {out }}$ is used to calculate the previously introduced leak-off $q_{L}$. It yields

$$
\partial_{t} h+\left(v_{1}, v_{2}\right) \cdot \nabla_{x_{1}, x_{2}} h-v_{3}=-v_{\text {out }} \quad \text { on } \quad x_{3}=h\left(x_{1}, x_{2}, t\right),
$$

and in the dimensionless form we have

$$
\partial_{t} w+\left(v_{x}^{\varepsilon}, v_{y}^{\varepsilon}\right) \cdot \nabla_{x, y} w-v_{z}^{\varepsilon}=-v_{L O} \quad \text { on } \quad z=w(x, y, t)
$$

where $v_{o u t}=U \varepsilon v_{L O}$. As in lubrication theory, we expand the velocities and the pressure as follows

$$
\begin{gathered}
v_{x}^{\varepsilon}=v_{x}^{0}+\varepsilon^{2} v_{x}^{1}+O\left(\varepsilon^{4}\right) ; \quad v_{y}^{\varepsilon}=v_{y}^{0}+\varepsilon^{2} v_{y}^{1}+O\left(\varepsilon^{4}\right) ; \quad v_{z}^{\varepsilon}=v_{z}^{0}+\varepsilon^{2} v_{z}^{1}+O\left(\varepsilon^{4}\right) ; \\
p^{\varepsilon}=p^{0}+\varepsilon^{2} p^{1}+O\left(\varepsilon^{4}\right) ; \\
w= \begin{cases}w^{(2)}(x, y, t) & \text { for } z \geq 0 \\
w^{(1)}(x, y, t) & \text { for } \quad z<0\end{cases}
\end{gathered}
$$

Inserting (50)-(52) into (44)-(47) yields at the order $O(1)$ in $\Omega_{F}$ :

$$
\begin{gathered}
-\partial_{x} p^{0}+\partial_{z z} v_{x}^{0}=0 ; \quad-\partial_{y} p^{0}+\partial_{z z} v_{y}^{0}=0 ; \quad-\partial_{z} p^{0}=0 \\
\partial_{x} v_{x}^{0}+\partial_{y} v_{y}^{0}+\partial_{z} v_{z}^{0}=0 ; \quad v_{x}^{0}(x, y, w, t)=0=v_{y}^{0}(x, y, w, t) .
\end{gathered}
$$


Following [43], we use (53)-(54) to calculate $v_{x}^{0}, v_{y}^{0}$ and obtain PDE for $p^{0}$ :

$$
\begin{gathered}
K(z)=\frac{1}{2}\left(w^{(2)}-z\right)\left(z-w^{(1)}\right), \quad v_{x}^{0}=-K \partial_{x} p^{0}, \quad v_{y}^{0}=-K \partial_{y} p^{0}, \\
\partial_{t} w=v_{z}^{0}(x, y, w)-v_{L O}, \\
K_{e}(z)=\int_{z}^{w^{(2)}} K(s) d s=\frac{\left(w^{(2)}-z\right)^{2}}{12}\left(w^{(2)}+2 z-3 w^{(1)}\right), \\
K^{a b s}=K_{e}\left(w^{(1)}\right)=\frac{\left(w^{(2)}-w^{(1)}\right)^{3}}{12}, \\
\partial_{t}\left(w^{(2)}-w^{(1)}\right)+v_{L O}^{(2)}-v_{L O}^{(1)}=\operatorname{div} \operatorname{din}_{x, y}\left(K^{a b s} \nabla_{x, y} p^{0}\right)= \\
K^{a b s} \Delta_{x, y} p^{0}+\nabla_{x, y} K^{a b s} \nabla_{x, y} p^{0} .
\end{gathered}
$$

The equation (59) is the Reynolds lubrication equation (see [43]). We note that this expansion is not valid in the fracture tip region and that the Navier-Stokes equation do not simplify there.

In order to have a Darcy-type law for the effective flow, we still miss the equation for $v_{z}^{0}$. After integrating the incompressibility relation (54) we obtain

$$
v_{z}^{0}(x, y, t)=-K_{e}(z) \Delta_{x, y} p^{0}-\nabla_{x, y} K_{e}(z) \nabla_{x, y} p^{0}+\partial_{t} w^{(2)}+v_{L O}^{(2)} .
$$

Next we use equation (59) to eliminate $\Delta_{x, y} p^{0}$. Insertion of the expression for it into (60) yields

$$
\begin{gathered}
v_{z}^{0}(x, y, t)=K_{e}(z) \nabla_{x, y} \log \frac{K^{a b s}}{K_{e}(z)} \cdot \nabla_{x, y} p^{0}+\left(1-\frac{K_{e}(z)}{K^{a b s}}\right)\left(\partial_{t} w^{(2)}+v_{L O}^{(2)}\right)+ \\
\frac{K_{e}(z)}{K^{a b s}}\left(\partial_{t} w^{(1)}+v_{L O}^{(1)}\right) .
\end{gathered}
$$

Hence we got the following Darcy's law

$$
\begin{aligned}
& {\left[\begin{array}{c}
v_{x}^{0} \\
v_{y}^{0} \\
v_{z}^{0}
\end{array}\right]=\left[\begin{array}{ccc}
-K(z) & 0 & K_{e}(z) \partial_{x} \log \frac{K^{a b s}}{K_{e}(z)} \\
0 & -K(z) & K_{e}(z) \partial_{y} \log \frac{K^{a b s}}{K_{e}(z)} \\
K_{e}(z) \partial_{x} \log \frac{K^{a b s}}{K_{e}(z)} & K_{e}(z) \partial_{y} \log \frac{K^{a b s}}{K_{e}(z)} & 0
\end{array}\right] \nabla_{x, y, z} p^{0}} \\
& +\left[\begin{array}{c}
0 \\
0 \\
\left(1-\frac{K_{e}(z)}{K^{a b s}}\right)\left(\partial_{t} w^{(2)}+v_{L O}^{(2)}\right)+\frac{K_{e}(z)}{K^{a b s}}\left(\partial_{t} w^{(1)}+v_{L O}^{(1)}\right)
\end{array}\right] .
\end{aligned}
$$

Darcy's law (62) is supplemented by the equation $\partial_{z} p^{0}=0$. In fact the dependence on $z$ for the effective pressure comes only at order $O\left(\varepsilon^{2}\right)$. Hence, since Darcy's law (62) is valid at order $O\left(\varepsilon^{2}\right)$, we can include the equation $\partial_{z} p^{0}=0$ into it by putting an $O\left(\varepsilon^{-2}\right)$ coefficient at the place $(3,3)$ in the permeability. This approach 
yields

$$
\begin{aligned}
& {\left[\begin{array}{c}
v_{x}^{0} \\
v_{y}^{0} \\
v_{z}^{0}
\end{array}\right]=\left[\begin{array}{ccc}
-K(z) & 0 & K_{e}(z) \partial_{x} \log \frac{K^{a b s}}{K_{e}(z)} \\
0 & -K(z) & K_{e}(z) \partial_{y} \log \frac{K^{a b s}}{K_{e}(z)} \\
K_{e}(z) \partial_{x} \log \frac{K^{a b s}}{K_{e}(z)} & K_{e}(z) \partial_{y} \log \frac{K^{a b s}}{K_{e}(z)} & -\frac{K(z)}{\varepsilon^{2}}
\end{array}\right] \nabla_{x, y, z} p^{0}} \\
& +\left[\begin{array}{c}
0 \\
0 \\
\left(1-\frac{K_{e}(z)}{K^{a b s}}\right)\left(\partial_{t} w^{(2)}+v_{L O}^{(2)}\right)+\frac{K_{e}(z)}{K^{a b s}}\left(\partial_{t} w^{(1)}+v_{L O}^{(1)}\right)
\end{array}\right] .
\end{aligned}
$$

In order to go back to the dimensional form, we set

$$
\begin{gathered}
K\left(x_{3}\right)=K(x, t)=\frac{1}{2}\left(h^{(2)}\left(x_{1}, x_{2}, t\right)-x_{3}\right)\left(x_{3}-h^{(1)}\left(x_{1}, x_{2}, t\right)\right) ; \\
K^{a b s}=K^{a b s}\left(x_{1}, x_{2}, t\right)=K_{e}\left(h^{(1)}\right)=\frac{\left(h^{(2)}\left(x_{1}, x_{2}, t\right)-h^{(1)}\left(x_{1}, x_{2}, t\right)\right)^{3}}{12} \\
\left.K_{e}\left(x_{3}\right)=K_{e}(x, t)\right)=\frac{\left(h^{(2)}\left(x_{1}, x_{2}, t\right)-x_{3}\right)^{2}}{12}\left(h^{(2)}\left(x_{1}, x_{2}, t\right)+2 x_{3}-3 h^{(1)}\left(x_{1}, x_{2}, t\right)\right) .
\end{gathered}
$$

Then (63) becomes

$$
\begin{aligned}
& {\left[\begin{array}{c}
v_{1}^{F} \\
v_{2}^{F} \\
v_{3}^{F}
\end{array}\right]=\left[\begin{array}{ccc}
-K\left(x_{3}\right) & 0 & K_{e}\left(x_{3}\right) \partial_{x_{1}} \log \frac{K^{a b s}}{K_{e}\left(x_{3}\right)} \\
0 & -K\left(x_{3}\right) & K_{e}\left(x_{3}\right) \partial_{x_{2}} \log \frac{K^{a b s}}{K_{e}\left(x_{3}\right)} \\
K_{e}\left(x_{3}\right) \partial_{x_{1}} \log \frac{K^{a b s}}{K_{e}\left(x_{3}\right)} & K_{e}\left(x_{3}\right) \partial_{x_{2}} \log \frac{K^{a b s}}{K_{e}\left(x_{3}\right)} & -K\left(x_{3}\right)
\end{array}\right] \frac{\nabla_{x} p^{F}}{\eta_{F}}} \\
& +\left[\begin{array}{c}
0 \\
0 \\
\left(1-\frac{K_{e}\left(x_{3}\right)}{K^{a b s}}\right)\left(\partial_{t} h^{(2)}+v_{\text {out }}^{(2)}\right)+\frac{K_{e}\left(x_{3}\right)}{K^{a b s}}\left(\partial_{t} h^{(1)}+v_{\text {out }}^{(1)}\right)
\end{array}\right] .
\end{aligned}
$$

Therefore, the fracture permeability is

$$
K_{F}=\left[\begin{array}{ccc}
-K\left(x_{3}\right) & 0 & K_{e}\left(x_{3}\right) \partial_{x_{1}} \log \frac{K^{a b s}}{K_{e}\left(x_{3}\right)} \\
0 & -K\left(x_{3}\right) & K_{e}\left(x_{3}\right) \partial_{x_{2}} \log \frac{K^{a b s}}{K_{e}\left(x_{3}\right)} \\
K_{e}\left(x_{3}\right) \partial_{x_{1}} \log \frac{K^{a b s}}{K_{e}\left(x_{3}\right)} & K_{e}\left(x_{3}\right) \partial_{x_{2}} \log \frac{K^{a b s}}{K_{e}\left(x_{3}\right)} & -K\left(x_{3}\right)
\end{array}\right]
$$

In the tip zone the permeability degenerates and Darcy's law (67) should not be used; we refer to Remark 2.2.

\section{Acknowledgments}

A.M. was partially supported by Conoco Phillips under Contract No. UTA10-000444, StatOil, and by The J. Tinsley Oden Faculty Fellowship Research Program. He would like to thank Center for Subsurface Modeling, Institute for Computational Engineering and Science (ICES), UT Austin for hospitality. The research by M. F. Wheeler was partially supported by the U.S. Department of Energy, Office of Science, Office of Basic Energy Sciences through DOE Modeling and Simulation of coupled complex multiscale subsurface phenomena under Contract No. DE-FG02-04ER25617, MOD. 010. T. Wick was partially supported by Conoco Phillips under Contract No. UTA10-000444, an ICES Postdoctoral Fellowship and the Humboldt foundation. 
Fluid-filled fractures in heterogeneous porous media

\section{References}

[1] W. Bangerth, T. Heister, G. Kanschat, And many others, Differential Equations Analysis Library, 2012.

[2] M.A. Bıот, Consolidation settlement under a rectangular load distribution, J. Appl. Phys., 12 (1941), pp. $426-430$.

[3] _ General theory of three-dimensional consolidation, J. Appl. Phys., 12 (1941), pp. 155-164.

[4] — Theory of elasticity and consolidation for a porous anisotropic solid, J. Appl. Phys., 25 (1955), pp. $182-185$.

[5] M. J. Borden, C. V. Verhoosel, M. A Scott, T. J. R. Hughes, And C. M. Landis, A phase-field description of dynamic brittle fracture, Comput. Meth. Appl. Mech. Engrg., 217 (2012), pp. 77-95.

[6] B. Bourdin, C. Chukwudozie, And K. Yoshioka, A variational approach to the numerical simulation of hydraulic fracturing. SPE Journal, Conference Paper 159154-MS, 2012.

[7] B. Bourdin, G.A. Francfort, And J.-J. Marigo, Numerical experiments in revisited brittle fracture, J. Mech. Phys. Solids, 48 (2000), pp. 797-826.

[8] _ _ The variational approach to fracture, J. Elasticity, 91 (2008), pp. 1-148.

[9] S. BURKe, C. ORTNER, AND E. SÜLI, An adaptive finite element approximation of a generalized Ambrosio-Tortorelli functional, Mathematical Models and Methods in Applied Sciences, 23 (2013), pp. 1663-1697.

[10] M. Hofacker C. Miehe, F. WelsChInger, Thermodynamically consistent phase-field models of fracture: variational principles and multi-field fe implementations, International Journal of Numerical Methods in Engineering, 83 (2013), pp. 1273-1311.

[11] B. CARRIER AND S. GRANET, Numerical modeling of hydraulic fracture problem in permeable medium using cohesive zone model, Engineering fracture mechanics, 79 (2012), pp. 312-328.

[12] H.-Y. Chen, L.W. Teufel, AND R.L. LEe, Coupled fluid flow and geomechanics in reservoir study i. theory and governing equations. SPE Journal, Conference Paper 30752, 1995.

[13] Z. Chen, G. HuAn, AND Y. MA, Computational Methods for Multiphase Flows in Porous Media, SIAM, 2006.

[14] O. Coussy, Mechanics of Porous Continua, Wiley, 1995.

[15] _ Poromechanics, Wiley, 2004.

[16] T. A. DAVIS AND I. S. DUFF, An unsymmetric-pattern multifrontal method for sparse LU factorization, SIAM J. Matrix Anal. Appl., 18 (1997), pp. 140-158.

[17] R. H. DEAN AND J.H. SCHMIDT, Hydraulic fracture predictions with a fully coupled geomechanical reservoir simulator. SPE Journal, Conference Paper 116470-MS, 2008.

[18] J. Desroches, E. Detournay, B. Lenoach, P. Papanastasiou, J.R.A. Pearson, M. Thiercelin, AND A. Cheng, The crack tip region in hydraulic fracturing, Proc. R. Soc. Lond., 67 (1994), p. 447. 
Mikelić, Wheeler, Wick

[19] E. Detournay And A. Peirce, On the moving boundary conditions for a hydraulic fracture. Preprint - online, 2014.

[20] G. A. Francfort AND J.-J. MARIGO, Revisiting brittle fracture as an energy minimization problem, J. Mech. Phys. Solids, 46 (1998), pp. 1319-1342.

[21] X. GAI, A coupled geomechanics and reservoir flow model on parallel computers, $\mathrm{PhD}$ thesis, The University of Texas at Austin, 2004.

[22] B. Ganis, V. Girault, M. Mear, G. Singh, And M.F. Wheeler, Modeling fractures in a poroelastic medium, Tech. Report ICES REPORT 13-09, The University of Texas at Austin, 2013.

[23] B. Ganis, M. E. Mear, A. Sakhaee-Pour, M. F. Wheeler, And T. Wick, Modeling fluid injection in fractures with a reservoir simulator coupled to a boundary element method, Tech. Report doi 10.1007/s10596-013-9396-5, Comput. Geosci., 2014.

[24] D.I. Garagash And E. Detournay, The tip region of a fluid-driven fracture in an elastic medium, ASME J. Appl. Mech., 67 (2000), pp. 183-192.

[25] A.A. GRIfFITH, The phenomena of rupture and flow in solids., Philos. Trans. R. Soc. Lond., 221 (1921), pp. 163-198.

[26] P. GuPTA AND C.A. DUARTE, Simulation of non-planar three-dimensional hydraulic fracture propagation, Int. J. Numer. Anal. Meth. Geomech., 38 (2014), pp. 1397-1430.

[27] K. M.D. HAls AND I. BerRe, Interaction between injection points during hydraulic fracturing, Tech. Report 1205.6299v1, arxiv, 2012.

[28] T. HeIsTER, M.F. WheEleR, AND T. WICK, A primal-dual active set method and predictor-corrector mesh adaptivity for computing fracture propagation using a phase-field approach, tech. report, 2014. ICES preprint 14-27, https://www.ices.utexas.edu/research/reports/.

[29] F. Irzal, J. J.C. Remmers, J. M. Huyghe, AND R. DE Borst, A large deformation formulation for fluid flow in a progressively fracturing porous material, Comput. Meth. Appl. Mech. Engrg., 256 (2013), pp. 29-37.

[30] A. Katiyar, J.T. Foster, H. Ouchi, And M.M. Sharma, A peridynamic formulation of pressure driven convective fluid transport in porous media, Journal of Computational Physics, 261 (2014), pp. 209 229.

[31] Y. Kovalyshen, Fluid-driven fracture in poroelastic medium, $\mathrm{PhD}$ thesis, The University of Minnesota, 2010.

[32] O.A. LAdyZhenskaja, V.A. Solonnikov, AND N.N. URALCEVA, Linear and quasi-linear equations of parabolic type, Translations of mathematical monographs, AMS Vol. 23, 1968.

[33] B. LeCAMPION AND E. Detournay, An implicit algorithm for the propagation of hydraulic fracture with a fluid lag, Comput. Meth. Appl. Mech. Engrg., 196 (2007), pp. 4863-4880.

[34] A. Mikelić, B. WANG, AND M.F. WheEleR, Numerical convergence study of iterative coupling for coupled flow and geomechanics. Comp. Geosci., doi: 10.1007/s10596-013-9393-8, 2014.

[35] A. Mikelić, M.F. WheEleR, AND T. WICK, A phase-field approach to the fluid filled fracture surrounded by a poroelastic medium. ICES-Preprint 13-15, Jun 2013. 
[36] — A quasi-static phase-field approach to the fluid filled fracture. ICES-Preprint, submitted for publication, Aug 2013.

[37] _ Phase-field modeling of pressurized fractures in a poroelastic medium. ICES-Preprint 14-18, submitted for publication, Jul 2014.

[38] A. Mikelić AND M. F. WhEELER, Convergence of iterative coupling for coupled flow and geomechanics, Comput Geosci, 17 (2012), pp. 455-462.

[39] S.L. Mitchell, R. Kuske, AND A.P. PeIRCE, An asymptotic framework for finite hydraulic fractures including leak-off, SIAM J. Appl. Math., 67 (2007), pp. 364-386.

[40] B. A. Schrefler, St. Secchi, ANd L. Simoni, On adaptive refinement techniques in multi-field problems including cohesive fracture, Comput. Methods Appl. Mech. Engrg., 195 (2006), pp. 444-461.

[41] _ On adaptive refinement techniques in multi-field problems including cohesive fracture, Comput. Meth. Appl. Mech. Engrg., 195 (2006), pp. 444-461.

[42] A SETTARI AND D A WALTERS, Advances in coupled geomechanical and reservoir modeling with applications to reservoir compaction, SPE Journal, 6 (2001), pp. 334-342.

[43] A. Z. SzERI, Fluid film lubrication, vol. 2nd edition, Cambridge University Press, 2011.

[44] M. WANGEn, Finite element modeling of hydraulic fracturing on a reservoir scale in $2 d$, Journal of Petroleum Science and Engineering, 77 (2011), pp. 274-285.

[45] _ Finite element modeling of hydraulic fracturing in 3d, Comput. Geosci., (2013).

[46] M.F. WHEELER, T. WICK, AND W. WOLLNER, An augmented-Lagangrian method for the phase-field approach for pressurized fractures, Comp. Meth. Appl. Mech. Engrg., 271 (2014), pp. 69-85.

[47] T. WICK, Solving monolithic fluid-structure interaction problems in arbitrary Lagrangian Eulerian coordinates with the deal.ii library, Archive of Numerical Software, 1 (2013), pp. 1-19.

[48] T. WICK, G. SINGH, AND M.F. WheELER, Pressurized fracture propagation using a phase-field approach coupled to a reservoir simulator. SPE 168597-MS, SPE Proc., 2013. 